\title{
Pseudodifferential operators on non-quasianalytic classes of Beurling type
}

\author{
by \\ C. Fernández, A. Galbis and D. Jornet (Valencia)
}

\begin{abstract}
We introduce pseudodifferential operators (of infinite order) in the framework of non-quasianalytic classes of Beurling type. We prove that such an operator with (distributional) kernel in a given Beurling class $\mathcal{D}_{(\omega)}^{\prime}$ is pseudo-local and can be locally decomposed, modulo a smoothing operator, as the composition of a pseudodifferential operator of finite order and an ultradifferential operator with constant coefficients in the sense of Komatsu, both operators with kernel in the same class $\mathcal{D}_{(\omega)}^{\prime}$. We also develop the corresponding symbolic calculus.
\end{abstract}

0. Introduction. The theory of pseudodifferential operators grew out of the study of singular integral operators, and developed after 1965 with the systematic studies of Kohn-Nirenberg [18], Hörmander [14] and others.

The study of several problems in classes of (non-quasianalytic) ultradifferentiable functions has also received much attention recently. These are intermediate classes between real analytic functions and $C^{\infty}$ functions. There are essentially two ways to introduce them: the theory of Komatsu [16], in which one looks at the growth of the derivatives on compact sets, and the theory developed by Björk [2] in 1966, following the ideas previously announced by Beurling, in which one pays attention to the growth of the Fourier transforms. We will work with ultradifferentiable functions as defined by Braun, Meise and Taylor [8]. Their point of view permits a unified treatment of both theories, contains the most relevant cases of Komatsu's theory and is strictly broader than Beurling-Björk's.

Pseudodifferential operators (of finite or infinite order) on Gevrey classes have been extensively studied by many authors ([5], [6], [15], [20], [25] among others). We refer to [23] for an excellent introduction to this topic. For more general classes of ultradifferentiable functions, following the approach of

2000 Mathematics Subject Classification: 46F05, 47G30, 35S05, 46E10.

Key words and phrases: pseudodifferential operator, ultradistribution, nonquasianalytic.

This research was supported by MCYT and FEDER proyecto n. BFM 2001-2670 and it is part of the Ph.D. thesis of D. Jornet. 
Komatsu, we refer to [21]. All of them deal with spaces of Roumieu type. These are spaces with a topological structure similar to that of the space of real analytic functions.

The purpose of this paper is to introduce pseudodifferential operators (p.d.o.) in the framework of ultradifferentiable functions of Beurling type, that is, spaces whose topology looks like the one of $C^{\infty}$. Our aim is to establish the basic theory in order to be able to face in the future topics like for instance hypoellipticity, Fourier integral operators, etc. As in [9], the pseudodifferential operators of $(\omega)$-class are defined as limits of operators with kernel in $\mathcal{E}_{(\omega)}(\Omega \times \Omega)$. With this point of view, it is immediate that the class of pseudodifferential operators is closed under taking adjoints and that every p.d.o. of $(\omega)$-class admits a continuous linear extension $A: \mathcal{E}_{(\omega)}^{\prime}(\Omega) \rightarrow \mathcal{D}_{(\omega)}^{\prime}(\Omega)$. We prove that such an operator shrinks $(\omega)$-singular supports (Theorem 2.18). Many operators are pseudodifferential operators according to our definition. In particular, we mention the linear partial differential operators with variable coefficients in a suitable class of functions, the $(\omega)$-smoothing operators and the ultradifferential operators in the sense of Komatsu. The convolution operator with an elementary solution of a given elliptic ultradifferential operator with constant coefficients is also a pseudodifferential operator. However, not every convolution operator is a p.d.o.

Since the class of p.d.o. also has to be closed under products of operators, and we need to express this property in terms of symbols, we develop the symbolic calculus.

The class of pseudodifferential operators of $(\omega)$-class contains the $(\omega)$ smoothing operators, operators of finite order and ultradifferential operators of $(\omega)$-class, and, as a consequence of 2.14 and 3.13, every pseudodifferential operator of $(\omega)$-class can be locally expressed, up to an $(\omega)$-smoothing operator, as the composition of an ultradifferential operator of $(\omega)$-class with constant coefficients and a p.d.o. of $(\omega)$-class and finite order. As far as we know there is no similar result in the Gevrey (Roumieu) setting.

1. Notation and preliminaries. In this section we introduce the relevant classes of functions and classes of amplitudes/symbols, and we establish some preliminary lemmata.

DEFINITION $1.1([8])$. A weight function is an increasing continuous function $\omega:[0, \infty[\rightarrow[0, \infty[$ with the following properties:

$(\alpha)$ there exists $L \geq 0$ with $\omega(2 t) \leq L(\omega(t)+1)$ for all $t \geq 0$,

( $\beta) \int_{1}^{\infty}\left(\omega(t) / t^{2}\right) d t<\infty$,

$(\gamma) \log (t)=o(\omega(t))$ as $t$ tends to $\infty$,

( $\delta) \varphi: t \mapsto \omega\left(e^{t}\right)$ is convex. 
For $z \in \mathbb{C}^{p}$ we put $\omega(z):=\omega(|z|)$, where $|z|:=\sup \left|z_{k}\right|$. The Young conjugate $\varphi^{*}:\left[0, \infty\left[\rightarrow \mathbb{R}\right.\right.$ of $\varphi$ is given by $\varphi^{*}(s):=\sup \{s t-\varphi(t): t \geq 0\}$. Here $\varphi$ is related to $\omega$ via Definition $1.1(\delta)$.

There is no loss of generality to assume that $\omega$ vanishes on $[0,1]$. Then $\varphi^{*}$ has only non-negative values, it is convex, $\varphi^{*}(t) / t$ is increasing and tends to $\infty$ as $t \rightarrow \infty$, and $\varphi^{* *}=\varphi$. We refer to [8] for properties of $\varphi^{*}$. Moreover, we assume that $\log t \leq \omega(t)$ for all $t>0$.

Definition $1.2([8])$. Let $\omega$ be a weight function. For an open set $\Omega \subset$ $\mathbb{R}^{p}$ we let

$$
\begin{array}{r}
\mathcal{E}_{(\omega)}(\Omega):=\left\{f \in C^{\infty}(\Omega):|f|_{K, \lambda}<\infty \text { for every } \lambda>0,\right. \\
\text { and every } K \subset \Omega \text { compact }\},
\end{array}
$$

where

$$
|f|_{K, \lambda}:=\sup _{x \in K} \sup _{\alpha \in \mathbb{N}_{0}^{p}}\left|f^{(\alpha)}(x)\right| \exp \left(-\lambda \varphi^{*}(|\alpha| / \lambda)\right) .
$$

$\mathcal{E}_{(\omega)}(\Omega)$ carries the metric locally convex topology given by the sequence of seminorms $|f|_{K_{n}, \lambda_{n}}$, where $\left(K_{n}\right)$ is any compact exhaustion of $\Omega$ and $\left(\lambda_{n}\right)$ is any increasing and unbounded sequence of positive numbers.

For $K \subset \Omega$ compact, we denote $\mathcal{E}_{(\omega)}(\Omega) \cap \mathcal{D}(K)$ by $\mathcal{D}_{(\omega)}(K)$. For $f \in$ $\mathcal{D}_{(\omega)}(K)$ we put $|f|_{\lambda}:=|f|_{K, \lambda}$. Then $\mathcal{D}_{(\omega)}(\Omega)=\operatorname{ind}_{n} \mathcal{D}_{(\omega)}\left(K_{n}\right)$, where $\left(K_{n}\right)$ is any compact exhaustion of $\Omega$. The elements of $\mathcal{D}_{(\omega)}^{\prime}(\Omega)$ are called ultradistributions of Beurling type.

The space $\mathcal{D}_{L_{1},(\omega)}\left(\mathbb{R}^{p}\right)$ is the set of all $C^{\infty}$ functions $f$ on $\mathbb{R}^{p}$ such that $\|f\|_{1, n}<\infty$ for each $n \in \mathbb{N}$, where

$$
\|f\|_{1, n}:=\sup _{\alpha \in \mathbb{N}_{0}^{p}}\left\|f^{(\alpha)}\right\|_{L_{1}} \exp \left(-n \varphi^{*}(|\alpha| / n)\right) .
$$

The inclusions $\mathcal{D}_{(\omega)}\left(\mathbb{R}^{p}\right) \subset \mathcal{D}_{L_{1},(\omega)}\left(\mathbb{R}^{p}\right) \subset \mathcal{E}_{(\omega)}\left(\mathbb{R}^{p}\right)$ are continuous and have dense range.

We start with some elementary but useful properties of $\varphi^{*}$ that follow from the convexity of $\varphi^{*}$ and the fact that $\varphi^{*}(0)=0$.

Lemma 1.3. (1) For every $\lambda, s, t>0$ we have

$$
2 \lambda \varphi^{*}\left(\frac{s+t}{2 \lambda}\right) \leq \lambda \varphi^{*}\left(\frac{s}{\lambda}\right)+\lambda \varphi^{*}\left(\frac{t}{\lambda}\right) \leq \lambda \varphi^{*}\left(\frac{s+t}{\lambda}\right) .
$$

(2) Let $L \in \mathbb{N}$ be such that $\omega($ et $) \leq L(1+\omega(t))$. Then

$$
k t+L^{k} \varphi^{*}\left(\frac{t}{L^{k}}\right) \leq \varphi^{*}(t)+\sum_{j=1}^{k} L^{j}
$$

for all $t \geq 0$ and $k \in \mathbb{N}$. 
Let $L \in \mathbb{N}$ be such that $\omega(e t) \leq L(1+\omega(t))$. Then $|\alpha|+n L \varphi^{*}(|\alpha| / n L) \leq$ $n L+n \varphi^{*}(|\alpha| / n)$. Therefore, if

$$
q_{K, n}(f):=\sup _{x \in K} \sup _{\alpha \in \mathbb{N}_{0}^{p}} e^{-|\alpha|}\left|f^{(\alpha)}(x)\right| \exp \left(-n \varphi^{*}(|\alpha| / n)\right)
$$

then

$$
q_{K, n}(f) \leq|f|_{K, n} \leq e^{n L} q_{K, n L}(f),
$$

so the topology of $\mathcal{E}_{(\omega)}(\Omega)$ can also be described by the system of seminorms $\left\{q_{K, n}\right\}$.

Lemma 1.4. For every $n, k \in \mathbb{N}$ and $t \geq 1$ we have

(1) $t^{k} \leq e^{n \varphi^{*}(k / n)} e^{n \omega(t)}$,

(2) $\inf _{j \in \mathbb{N}_{0}} t^{-j} e^{k \varphi^{*}(j / k)} \leq e^{-k \omega(t)+\log t}$.

The following result permits us to split $\mathbb{R}$ into intervals in which the infimum in 1.4 is attained in a finite set.

Lemma 1.5. Fix $k, N \in \mathbb{N}$ and assume

$$
\frac{k}{N} \varphi^{*}\left(\frac{N}{k}\right) \leq \log t<\frac{k}{N+1} \varphi^{*}\left(\frac{N+1}{k}\right) .
$$

Then

(1) $\min _{0 \leq j \leq N} t^{-j} e^{k \varphi^{*}(j / k)} \leq e^{-k \omega(t)+\log t}$,

(2) $t^{-N} e^{2 k \varphi^{*}(N / 2 k)} \leq e^{-k \omega(t)+\log t}$.

Proof. (1) Since $\varphi^{*}(t) / t$ is increasing, we have $\log t<(k / j) \varphi^{*}(j / k)$ (and so $\left.t^{-j} e^{k \varphi^{*}(j / k)}>1\right)$ for every $j \geq N+1$. Now the conclusion follows from Lemma 1.4.

(2) We already know that $t^{-(N-l)} e^{k \varphi^{*}((N-l) / k)} \leq e^{-k \omega(t)+\log t}$ for some $l=0,1, \ldots, N$ (see 1.4). Then, using the inequality $(k / l) \varphi^{*}(l / k) \leq \log t$, we obtain

$$
t^{-N} e^{2 k \varphi^{*}(N / 2 k)} \leq t^{-(N-l)} t^{-l} e^{k \varphi^{*}((N-l) / k)} e^{k \varphi^{*}(l / k)} \leq e^{-k \omega(t)+\log t} .
$$

The definition of symbol in $[13,23,25]$ motivates our next definition. As we will check in 2.11 , for the limit case $\omega(t)=\log (1+t)$ we recover the symbols in [13], whereas for the Gevrey weights $\omega(t)=t^{d}, 0<d<1$, our definition is what can be reasonably expected if one translates to the Beurling setting the definition of symbol of Gevrey class (Roumieu setting) in $[23,25]$. The introduction of symbols of type $(\varrho, \delta)$ perhaps makes the reading of the paper uncomfortable but it seems convenient for the construction of parametrices of hypoelliptic operators. See Proposition 2.12 .

Definition 1.6. Let $\Omega$ be an open set in $\mathbb{R}^{p}, 0 \leq \delta<\varrho \leq 1, d:=\varrho-\delta$ and assume that $\omega(t)=o\left(t^{d}\right)$ as $t \rightarrow \infty$. An amplitude in $S_{\varrho, \delta}^{m, \omega}(\Omega)$ is a 
function $a(x, y, \xi)$ in $C^{\infty}\left(\Omega \times \Omega \times \mathbb{R}^{p}\right)$ such that for every compact set $Q \subset \Omega \times \Omega$ there are $R \geq 1$ and a sequence $C_{n}>0, n \in \mathbb{N}$, with

(*) $\left|D_{x}^{\alpha} D_{y}^{\gamma} D_{\xi}^{\beta} a(x, y, \xi)\right| \leq C_{n} e^{(\varrho-\delta) n \varphi^{*}(|\alpha+\beta+\gamma| / n)} e^{m \omega(\xi)}(1+|\xi|)^{|\alpha+\gamma| \delta-|\beta| \varrho}$

for every $n \in \mathbb{N},(x, y) \in Q$, and $\xi$ with $\log (|\xi| / R) \geq(n /|\beta|) \varphi^{*}(|\beta| / n)$.

For $|\beta|=0$ the estimate holds for every $\xi \in \mathbb{R}^{p}$.

In the case $a(x, y, \xi)=p(x, \xi)$, the function $p(x, \xi)$ is usually called a symbol.

Since the function $\varphi^{*}$ is convex, we may replace $e^{(\varrho-\delta) n \varphi^{*}(|\alpha+\beta+\gamma| / n)}$ in (*) by $e^{(\varrho-\delta) n \varphi^{*}(|\alpha+\gamma| / n)+(\varrho-\delta) n \varphi^{*}(|\beta| / n)}$ (see Lemma 1.3).

Some examples will be given in 2.11 .

REMARK 1.7. We make some comments on the requirement $\omega(t)=o\left(t^{d}\right)$ in the definition of amplitude.

(1) If $\varrho=1$ and $\delta=0$, this does not mean any restriction on $\omega$. For other values of $\varrho$ and/or $\delta$, this extra assumption means that $\mathcal{E}_{(\omega)}(\Omega)$ contains, as a continuously and densely embedded subspace, the Gevrey class $\Gamma^{\{1 / d\}}(\Omega)$ and it ensures that $j !=O\left(e^{(\varrho-\delta) n \varphi^{*}(j / n)}\right)$ as $j \rightarrow \infty$ for every $n \in \mathbb{N}$. As shown in [22], if $\omega$ is a weight function for which an extension of the classical Borel theorem holds, then $\mathcal{E}_{(\omega)}(\Omega)$ contains $\Gamma^{\{s\}}(\Omega)$ for some $s>1$.

(2) If $\omega(t)=(\log (1+t))^{s}, s>1$, for $t$ large enough then $\omega(t)=o\left(t^{d}\right)$ for every $0<d<1$, whereas if $\omega(t)=t(\log t)^{-a}(a>1)$, then $\mathcal{E}_{(\omega)}(\Omega)$ does not contain any Gevrey class.

Lemma 1.8. Let $a(x, y, \xi)$ be an amplitude in $S_{\varrho, \delta}^{m, \omega}(\Omega)$. Then for every compact set $Q \subset \Omega \times \Omega$ there exists a sequence $C_{n}>0, n \in \mathbb{N}$, such that

$$
\left|D_{x}^{\alpha} D_{y}^{\gamma} a(x, y, \xi)\right| \leq C_{n} e^{(\varrho-\delta) n \varphi^{*}(|\alpha| / n)} e^{(\varrho-\delta) n \varphi^{*}(|\gamma| / n)}|\xi|^{\delta|\alpha+\gamma|} e^{m \omega(\xi)}
$$

for every $(x, y) \in Q$ and $|\xi| \geq 1$.

Proof. We put $B:=\left(2^{\delta}\right)^{1 /(\varrho-\delta)}$ and we take $k \in \mathbb{N}$ with $B \leq e^{k}$ and $L \in \mathbb{N}$ such that $\omega($ et $) \leq L(1+\omega(t))$ for all $t \geq 0$. Finally, we fix $n \in \mathbb{N}$ and we take $l:=2 n L^{k}$. According to Definition 1.6 there is $C>0$ such that for all $(x, y) \in Q$ and $|\xi| \geq 1$ we have

$$
\left|D_{x}^{\alpha} D_{y}^{\gamma} a(x, y, \xi)\right| \leq C e^{(\varrho-\delta) l \varphi^{*}(|\alpha+\gamma| / l)}(2|\xi|)^{\delta|\alpha+\gamma|} e^{m \omega(\xi)} .
$$

An application of Lemma 1.3 gives $k|\alpha+\gamma|+l \varphi^{*}(|\alpha+\gamma| / l) \leq A+n \varphi^{*}(|\alpha| / n)$ $+n \varphi^{*}(|\gamma| / n)$ for some constant $A>0$. Since $2^{\delta|\alpha+\gamma|} \leq e^{k(\varrho-\delta)|\alpha+\gamma|}$, we conclude that

$$
\left|D_{x}^{\alpha} D_{y}^{\gamma} a(x, y, \xi)\right| \leq C e^{A} e^{(\varrho-\delta) n \varphi^{*}(|\alpha| / n)} e^{(\varrho-\delta) n \varphi^{*}(|\gamma| / n)}|\xi|^{\delta|\alpha+\gamma|} e^{m \omega(\xi)} .
$$

Proposition 1.9. Let $a(x, y, \xi)$ be an amplitude in $S_{\varrho, \delta}^{m, \omega}(\Omega)$ and let $f \in \mathcal{D}_{(\omega)}(\Omega)$. For every compact set $K \subset \Omega$ and $n, \lambda \in \mathbb{N}$ there is a constant 
$C>0$ such that

$$
\left|\int D_{x}^{\alpha} a(x, y, \xi) f(y) e^{-i y \xi} d y\right| \leq C e^{-\lambda \omega(\xi)} e^{n \varphi^{*}(|\alpha| / n)}
$$

for every $x \in K, \xi \in \mathbb{R}^{p}$. Moreover, a similar estimate holds if we replace $a(x, y, \xi)$ by $b(x, y, \xi):=a(x, y, \xi) e^{i x \xi}$.

Proof. For every $s, n \in \mathbb{N}$ there is $C>0$ such that

$$
\left|D_{x}^{\alpha} D_{y}^{\gamma} a(x, y, \xi)\right| \leq C e^{(\varrho-\delta) s n \varphi^{*}(|\alpha| / s n)} e^{(\varrho-\delta) s n \varphi^{*}(|\gamma| / s n)}|\xi|^{\delta|\alpha+\gamma|} e^{m \omega(\xi)}
$$

for every $x \in K, y \in \operatorname{supp} f$ and $|\xi| \geq 1$. Since $\varphi^{*}(t) / t$ is increasing we get $(\varrho-\delta) s n \varphi^{*}(|\alpha| / s n) \leq(\varrho-\delta) n \varphi^{*}(|\alpha| / n)$. From 1.4 we deduce that $|\xi|^{\delta|\alpha|} e^{-\delta n \varphi^{*}(|\alpha| / n)} \leq e^{n \delta \omega(\xi)}$, which implies

$$
\left|D_{x}^{\alpha} D_{y}^{\gamma} a(x, y, \xi)\right| \leq C e^{\varrho n \varphi^{*}(|\alpha| / n)} e^{(\varrho-\delta) s n \varphi^{*}(|\gamma| / s n)}|\xi|^{\delta|\gamma|} e^{(m+n \delta) \omega(\xi)}
$$

for every $x \in K, y \in \operatorname{supp} f$ and $|\xi| \geq 1$.

We now fix $\xi \in \mathbb{R}^{p},|\xi| \geq 1$, and we take $1 \leq k \leq p$ with $|\xi|=\left|\xi_{k}\right|$. For every $j \in \mathbb{N}$ we have, after integrating by parts,

$$
\int D_{x}^{\alpha} a(x, y, \xi) f(y) e^{-i y \xi} d y=\frac{1}{\xi_{k}^{j}} \int D_{y_{k}}^{j}\left(D_{x}^{\alpha} a(x, y, \xi) f(y)\right) e^{-i y \xi} d y
$$

Hence, for every $j, s \in \mathbb{N}$ and some constant $C$ which only depends on $n, s$ and on the Lebesgue measure of the support of $f$, we have

$$
\begin{aligned}
& \left|\int D_{x}^{\alpha} a(x, y, \xi) f(y) e^{-i y \xi} d y\right| \\
& \quad \leq C|f|_{s n} e^{\varrho n \varphi^{*}(|\alpha| / n)} e^{(m+n \delta) \omega(\xi)} \sum_{l=0}^{j}\left(\begin{array}{c}
j \\
l
\end{array}\right) \frac{e^{(\varrho-\delta) s n \varphi^{*}(l / s n)}}{|\xi|^{l-\delta l}} \frac{e^{s n \varphi^{*}((j-l) / s n)}}{|\xi|^{j-l}} .
\end{aligned}
$$

Consider the natural number $N$ such that

$$
\frac{s n}{N} \varphi^{*}\left(\frac{N}{s n}\right) \leq \log \left(\frac{|\xi|}{2^{1 /(\varrho-\delta)}}\right)<\frac{s n}{N+1} \varphi^{*}\left(\frac{N+1}{s n}\right) .
$$

Then, for every $l<j \leq N$, we have $e^{\frac{s n}{j-l} \varphi^{*}((j-l) / s n)} \leq e^{\frac{s n}{N} \varphi^{*}(N / s n)} \leq|\xi|$, hence $e^{s n \varphi^{*}((j-l) / s n)} /|\xi|^{j-l} \leq 1$, which implies, since $0<\varrho-\delta \leq 1$,

$$
\frac{e^{s n \varphi^{*}((j-l) / s n)}}{|\xi|^{j-l}} \leq\left(\frac{e^{s n \varphi^{*}((j-l) / s n)}}{|\xi|^{j-l}}\right)^{\varrho-\delta}
$$

and

$$
\frac{e^{(\varrho-\delta) s n \varphi^{*}(l / s n)}}{|\xi|^{(\varrho-\delta) l}} \frac{e^{s n \varphi^{*}((j-l) / s n)}}{|\xi|^{j-l}} \leq\left(\frac{e^{s n \varphi^{*}(j / s n)}}{|\xi|^{j}}\right)^{\varrho-\delta}
$$

(see Lemma 1.3). We finally deduce 


$$
\begin{aligned}
& \left|\int D_{x}^{\alpha} a(x, y, \xi) f(y) e^{-i y \xi} d y\right| \\
& \leq C|f|_{s n} e^{(\delta n+m) \omega(\xi)} e^{n \varphi^{*}(|\alpha| / n)} \sum_{l=0}^{j}\left(\begin{array}{c}
j \\
l
\end{array}\right)\left(\frac{e^{s n \varphi^{*}(j / s n)}}{|\xi|^{j}}\right)^{\varrho-\delta} \\
& \leq C|f|_{s n} e^{(\delta n+m) \omega(\xi)} e^{n \varphi^{*}(|\alpha| / n)}\left(\frac{e^{s n \varphi^{*}(j / s n)}}{\left(|\xi| / 2^{1 /(\varrho-\delta)}\right)^{j}}\right)^{\varrho-\delta}
\end{aligned}
$$

for every $j \leq N$. It follows from Lemma 1.5 that

$$
\begin{aligned}
& \left|\int D_{x}^{\alpha} a(x, y, \xi) f(y) e^{-i y \xi} d y\right| \\
& \quad \leq C|f|_{s n} e^{(\delta n+m) \omega(\xi)} e^{n \varphi^{*}(|\alpha| / n)} e^{-s n(\varrho-\delta) \omega\left(|\xi| / 2^{1 /(\varrho-\delta)}\right)+\log \left(|\xi| / 2^{1 /(\varrho-\delta)}\right)} .
\end{aligned}
$$

Now it suffices to choose $s$ large enough. The corresponding estimate for $b(x, y, \xi)$ can be deduced with a similar argument.

2. Pseudodifferential operators. In this section we define pseudodifferential operators on non-quasianalytic classes of Beurling type. Our approach is as in [9], that is, pseudodifferential operators on $\mathcal{D}_{(\omega)}(\Omega)$ are obtained as limits of operators with kernels in $\mathcal{E}_{(\omega)}(\Omega \times \Omega)$. We examine several examples showing that the class of pseudodifferential operators contains enough elements and we show that they are pseudolocal.

It is easy to see from the definition of amplitude that $\{a(\cdot, \cdot, \xi):|\xi| \leq T\}$ is a bounded set in $\mathcal{E}_{(\omega)}(\Omega \times \Omega)$ for every $T>0$; hence we easily deduce

LEMmA 2.1. Let $a(x, y, \xi)$ be an amplitude in $S_{\varrho, \delta}^{m, \omega}(\Omega)$ and let $\Psi \in$ $\mathcal{D}_{(\omega)}\left(\mathbb{R}^{p}\right)$. Then

(1) $K(x, y):=\int a(x, y, \xi) e^{i(x-y) \xi} \Psi(\xi) d \xi$ belongs to $\mathcal{E}_{(\omega)}(\Omega \times \Omega)$,

$(2) B: \mathcal{D}_{(\omega)}(\Omega) \rightarrow \mathcal{E}_{(\omega)}(\Omega), B(f)(x):=\int K(x, y) f(y) d y$, is a continuous linear operator.

Let $\Psi \in \mathcal{D}_{(\omega)}\left(\mathbb{R}^{p}\right)$ be a test function such that $\Psi(\xi)=1$ for $|\xi| \leq 1$ and $\Psi(\xi)=0$ for $|\xi| \geq 2$. We put

$$
\left(A_{\delta} f\right)(x):=\iint a(x, y, \xi) e^{i(x-y) \xi} f(y) \Psi(\delta \xi) d y d \xi
$$

THEOREM 2.2. Let $a(x, y, \xi)$ be an amplitude in $S_{\varrho, \delta}^{m, \omega}(\Omega)$. Then

(1) For every $f \in \mathcal{D}_{(\omega)}(\Omega)$ the limit $A(f):=\mathcal{E}_{(\omega)}(\Omega)-\lim _{\delta \rightarrow 0+} A_{\delta}(f)$ exists and $A: \mathcal{D}_{(\omega)}(\Omega) \rightarrow \mathcal{E}_{(\omega)}(\Omega)$ is a continuous linear operator,

(2) $(A f)(x)=\int\left(\int a(x, y, \xi) e^{i(x-y) \xi} f(y) d y\right) d \xi$.

Proof. (1) We fix a compact set $K \subset \Omega$ and $n \in \mathbb{N}$. We put

$$
I(x, \xi):=\int a(x, y, \xi) f(y) e^{i(x-y) \xi} d y
$$


and we apply Proposition 1.9 to get a constant $C>0$ such that

$$
\left|D_{x}^{\alpha} I(x, \xi)\right| e^{-n \varphi^{*}(|\alpha| / n)} \leq C e^{-\omega(\xi)}
$$

for every $x \in K, \alpha \in \mathbb{N}_{0}^{p}$ and $\xi \in \mathbb{R}^{p}$. Hence, for $0<\delta_{2}<\delta_{1}<1$ we can estimate

$$
q_{K, n}\left(A_{\delta_{1}} f-A_{\delta_{2}} f\right) \leq C \int_{|\xi| \geq 1 / \delta_{1}} e^{-\omega(\xi)}\left|\Psi\left(\delta_{1} \xi\right)-\Psi\left(\delta_{2} \xi\right)\right| d \xi,
$$

which implies that the limit $A(f):=\mathcal{E}_{(\omega)}(\Omega)-\lim _{\delta \rightarrow 0+} A_{\delta}(f)$ exists. An application of the uniform boundedness principle gives the continuity of $A: \mathcal{D}_{(\omega)}(\Omega) \rightarrow \mathcal{E}_{(\omega)}(\Omega)$.

(2) We observe that

$$
(A f)(x)=\lim _{n \rightarrow \infty} \int\left(\int a(x, y, \xi) f(y) e^{-i y \xi} d y\right) e^{i x \xi} \Psi(\xi / n) d \xi .
$$

Since for every $k \in \mathbb{N}$ there is $C>0$ such that $\left|\int a(x, y, \xi) f(y) e^{-i y \xi} d y\right| \leq$ $C e^{-k \omega(\xi)}$ for every $\xi \in \mathbb{R}^{p}$, we can apply the dominated convergence theorem to conclude that $(A f)(x)=\int\left(\int a(x, y, \xi) e^{i(x-y) \xi} f(y) d y\right) d \xi$.

Definition 2.3. The operator $A: \mathcal{D}_{(\omega)}(\Omega) \rightarrow \mathcal{E}_{(\omega)}(\Omega)$ introduced in Theorem 2.2 is called a pseudodifferential operator of $(\omega)$-class associated to the amplitude $a(x, y, \xi)$.

In the case $a(x, y, \xi)=p(x, \xi)$ the pseudodifferential operator $A$ is denoted by $P(x, D)$ and we have

$$
P(x, D) f=\int p(x, \xi) e^{i x \xi} \widehat{f}(\xi) d \xi
$$

for every $f \in \mathcal{D}_{(\omega)}(\Omega)$. It is clear that the expression above makes sense for $f \in \mathcal{D}_{(\omega)}\left(\mathbb{R}^{p}\right)$, and even for a wider class of functions.

Proposition 2.4. The operator $P(x, D)$ associated to a symbol $p(x, \xi)$ in $S_{\varrho, \delta}^{m, \omega}(\Omega)$ can be extended to $\mathcal{D}_{L_{1},(\omega)}\left(\mathbb{R}^{p}\right)$ and the extension is linear and continuous taking values in $\mathcal{E}_{(\omega)}(\Omega)$.

Proof. Given $f \in \mathcal{D}_{L_{1},(\omega)}\left(\mathbb{R}^{p}\right)$ and $k \in \mathbb{N}$, its Fourier transform satisfies

$$
\sup _{\xi \in \mathbb{R}^{p}}|\widehat{f}(\xi)| e^{k \omega(\xi)} \leq C\|f\|_{1, k+1}
$$

for some constant $C$ depending only on the weight $\omega$ (see [12, 1.1.23]). Hence, the integral above is also convergent for $f \in \mathcal{D}_{L_{1},(\omega)}\left(\mathbb{R}^{p}\right)$. Thus $P(x, D)$ can be extended to $\mathcal{D}_{L_{1},(\omega)}\left(\mathbb{R}^{p}\right)$, and the extension is linear.

Given a compact subset $K$ of $\Omega$ and $\alpha \in \mathbb{N}_{0}^{p}$,

$$
\left|D_{x}^{\alpha}(P(x, D) f)\right| \leq \sum_{\beta \leq \alpha}\left(\begin{array}{c}
\alpha \\
\beta
\end{array}\right) \int\left|\widehat{D^{\beta}} f(\xi)\right|\left|D_{x}^{\alpha-\beta} p(x, \xi)\right| d \xi .
$$


As in 1.9 , there is a sequence $\left(C_{n}\right)$ of constants such that

$$
\left|D_{x}^{\gamma} p(x, \xi)\right| \leq C_{n} e^{n \varphi^{*}(|\gamma| / n)} e^{(m+n \delta) \omega(\xi)}
$$

for every multi-index $\gamma$, each $x \in K$ and $\xi \in \mathbb{R}^{p}$. Moreover, we deduce from the properties of $\varphi^{*}$ that

$$
\sup _{\xi \in \mathbb{R}^{p}}\left|\widehat{D^{\beta}} f(\xi)\right| e^{(n+m) \omega(\xi)} \leq C\left\|D^{\beta} f\right\|_{1, n+m+1} \leq C\|f\|_{1,2 n+2 m+2} e^{n \varphi^{*}(|\beta| / n)} .
$$

Therefore,

$$
q_{K, n}(P(x, D) f) \leq C C_{n}\|f\|_{1,2 n+2 m+2} \int e^{-\omega(\xi)} d \xi
$$

for $n$ large enough, which finishes the proof.

TheOREM 2.5. The pseudodifferential operator $A$ associated to an amplitude $a(x, y, \xi)$ in $S_{\varrho, \delta}^{m, \omega}(\Omega)$ admits a continuous linear extension $\mathcal{E}_{(\omega)}^{\prime}(\Omega) \rightarrow$ $\mathcal{D}_{(\omega)}^{\prime}(\Omega)$.

Proof. We consider $b(x, y, \xi):=a(y, x,-\xi)$, which is an amplitude in $S_{\varrho, \delta}^{m, \omega}(\Omega)$, and we denote by $B: \mathcal{D}_{(\omega)}(\Omega) \rightarrow \mathcal{E}_{(\omega)}(\Omega)$ the associated pseudodifferential operator. We only have to show that the transpose $B^{t}$ is the desired extension of $A$. To do this, we put

$$
\left(B_{\delta} f\right)(x):=\iint b(x, y, \xi) e^{i(x-y) \xi} f(y) \Psi(\delta \xi) d y d \xi .
$$

Then it is easy to prove that $\int \varphi\left(B_{\delta} \Phi\right)=\int\left(A_{\delta} \varphi\right) \Phi$ and an application of Theorem 2.2(i) gives the conclusion.

Corollary 2.6. Let $A: \mathcal{D}_{(\omega)}(\Omega) \rightarrow \mathcal{E}_{(\omega)}(\Omega)$ be the pseudodifferential operator with amplitude $a(x, y, \xi)$. Then $\left.A^{t}\right|_{\mathcal{D}_{(\omega)}(\Omega)}: \mathcal{D}_{(\omega)}(\Omega) \rightarrow \mathcal{E}_{(\omega)}(\Omega)$ is a pseudodifferential operator with amplitude $a(y, x,-\xi)$.

Theorem 2.7. The extension $\bar{P}(x, D): \mathcal{E}_{(\omega)}^{\prime}(\Omega) \rightarrow \mathcal{D}_{(\omega)}^{\prime}(\Omega)$ of the pseudodifferential operator $P(x, D)$ is given by

$$
\langle\bar{P}(x, D) \mu, \psi\rangle=\int \widehat{\mu}(\xi)\left(\int e^{i x \xi} p(x, \xi) \psi(x) d x\right) d \xi .
$$

Proof. Since $\langle P(x, D) \varphi, \psi\rangle=\int \widehat{\varphi}(\xi)\left(\int e^{i x \xi} p(x, \xi) \psi(x) d x\right) d \xi$ for every $\varphi, \psi \in \mathcal{D}_{(\omega)}(\Omega)$, we only have to prove that $\bar{P}(x, D): \mathcal{E}_{(\omega)}^{\prime}(\Omega) \rightarrow \mathcal{D}_{(\omega)}^{\prime}(\Omega)$ is a well defined, continuous linear operator. To do this we first fix $\mu \in \mathcal{E}_{(\omega)}^{\prime}(\Omega)$ and we let $B$ be a bounded set in $\mathcal{D}_{(\omega)}(\Omega)$. By the Paley-Wiener theorem $([8,7.4])$ there are constants $A, D>0$ such that $|\widehat{\mu}(\xi)| \leq D e^{A \omega(\xi)}$ for every $\xi \in \mathbb{R}^{p}$. Now we take $k:=A+1$ and we apply (the proof of) Proposition 1.9 to find a constant $C>0$ such that $\left|\int e^{i x \xi} p(x, \xi) \psi(x) d x\right| \leq C e^{-k \omega(\xi)}$ for every $\psi \in B$. Then

$$
\left|\widehat{\mu}(\xi)\left(\int e^{i x \xi} p(x, \xi) \psi(x) d x\right)\right| \leq C D e^{-\omega(\xi)},
$$


which implies that $\bar{P}(x, D) \mu: \mathcal{D}_{(\omega)}(\Omega) \rightarrow \mathbb{C}$ is bounded on bounded sets and, consequently, $\bar{P}(x, D) \mu \in \mathcal{D}_{(\omega)}^{\prime}(\Omega)$. Moreover the estimates just obtained also show that $\bar{P}(x, D): \mathcal{E}_{(\omega)}^{\prime}(\Omega) \rightarrow \mathcal{D}_{(\omega)}^{\prime}(\Omega)$ transforms bounded sets in $\mathcal{E}_{(\omega)}^{\prime}(\Omega)$ into weakly bounded sets in $\mathcal{D}_{(\omega)}^{\prime}(\Omega)$, and we conclude that $\bar{P}(x, D)$ is continuous.

The correspondence between amplitudes and operators is not one-to-one, that is, two different amplitudes may define the same operator (see Example 2.11(4) below). The situation is much better for symbols.

Corollary 2.8. Let $p(x, \xi)$ and $q(x, \xi)$ be symbols in $S_{\varrho, \delta}^{m, \omega}\left(\mathbb{R}^{p}\right)$ defining the same pseudodifferential operator. Then $p(x, \xi)=q(x, \xi)$.

Proof. We put $r(x, \xi):=p(x, \xi)-q(x, \xi)$. By hypothesis $R(x, D)$ is the null operator and hence $\left\langle\bar{R}(x, D) \delta_{y}, \psi\right\rangle=0$ for every $y \in \mathbb{R}^{p}$ and $\psi \in$ $\mathcal{D}_{(\omega)}\left(\mathbb{R}^{p}\right)$. For a fixed $\psi \in \mathcal{D}_{(\omega)}(\Omega)$ we set $I(\xi):=\int e^{i x \xi} r(x, \xi) \psi(x) d x$ and we deduce from Theorem 2.7 that the Fourier transform of the continuous function $I(\xi)$ vanishes. It follows that $\int e^{i x \xi} r(x, \xi) \psi(x) d x=0$ for every $\psi \in \mathcal{D}_{(\omega)}\left(\mathbb{R}^{p}\right)$, and so $r(x, \xi)=0$ everywhere.

In some cases it is possible to recover the symbol from the pseudodifferential operator it defines.

Proposition 2.9. Let $p(x, \xi)$ a symbol in $S_{\varrho, \delta}^{m, \omega}\left(\mathbb{R}^{p}\right)$ and assume that $P(x, D)$ admits a continuous linear extension $A: \mathcal{E}_{(\omega)}\left(\mathbb{R}^{p}\right) \rightarrow \mathcal{E}_{(\omega)}\left(\mathbb{R}^{p}\right)$. Then

$$
p(x, \xi)=\frac{1}{(2 \pi)^{p}} e^{-i x \xi} A\left(e^{i(\cdot) \xi}\right)(x) .
$$

Proof. Since $A^{t}: \mathcal{E}_{(\omega)}^{\prime}\left(\mathbb{R}^{p}\right) \rightarrow \mathcal{E}_{(\omega)}^{\prime}\left(\mathbb{R}^{p}\right)$ we see from 2.6 that $\left.A^{t}\right|_{\mathcal{D}_{(\omega)}\left(\mathbb{R}^{p}\right)}$ : $\mathcal{D}_{(\omega)}\left(\mathbb{R}^{p}\right) \rightarrow \mathcal{D}_{(\omega)}\left(\mathbb{R}^{p}\right)$ is a pseudodifferential operator defined by $b(x, y, \xi)$ $:=p(y,-\xi)$. Then for every $\varphi \in \mathcal{D}_{(\omega)}\left(\mathbb{R}^{p}\right)$ we obtain

$$
\left(A^{t} \varphi\right)(x)=\int e^{i x \xi}\left(\int p(y,-\xi) \varphi(y) e^{-i y \xi} d y\right) d \xi .
$$

We put $I(\xi):=\int p(y,-\xi) \varphi(y) e^{-i y \xi} d y$. Then $I \in L_{1}$ and moreover $\widehat{I}(-x)=$ $\left(A^{t} \varphi\right)(x)$, which implies, in particular, that $\widehat{I} \in \mathcal{D}_{(\omega)}\left(\mathbb{R}^{p}\right)$. Hence

$$
\left\langle A\left(e^{i(.) \xi}\right), \varphi\right\rangle=\int e^{i x \xi} \widehat{I}(-x) d x=(2 \pi)^{p} I(-\xi)=(2 \pi)^{p} \int p(x, \xi) \varphi(x) e^{i x \xi} d x,
$$

which finishes the proof.

In most of the forthcoming results we will need stronger conditions on the amplitude.

Definition 2.10. Let $\Omega$ be an open set in $\mathbb{R}^{p}, 0 \leq \delta<\varrho \leq 1, d:=\varrho-\delta$ and assume $\omega(t)=o\left(t^{d}\right)$ as $t \rightarrow \infty$. An amplitude in $\bar{A} S_{\varrho, \delta}^{m, \omega}(\Omega)$ is a function 
$a(x, y, \xi)$ in $C^{\infty}\left(\Omega \times \Omega \times \mathbb{R}^{p}\right)$ such that for every compact set $Q \subset \Omega \times \Omega$ there are $R, B \geq 1$ and a sequence $C_{n}>0, n \in \mathbb{N}$, with

$$
\left|D_{x}^{\alpha} D_{y}^{\gamma} D_{\xi}^{\beta} a(x, y, \xi)\right| \leq C_{n} B^{\beta} \beta ! e^{(\varrho-\delta) n \varphi^{*}(|\alpha+\gamma| / n)} e^{m \omega(\xi)}(1+|\xi|)^{|\alpha+\gamma| \delta-|\beta| \varrho}
$$

for every $n \in \mathbb{N},(x, y) \in Q$, and $\xi$ with $\log (|\xi| / R) \geq(n /|\beta|) \varphi^{*}(|\beta| / n)$.

An amplitude in $A S_{o, \delta}^{m, \omega}(\Omega)$ is said to be of finite order if it satisfies the inequalities above with $(1+|\xi|)^{m}$ instead of $e^{m \omega(\xi)}$.

It follows from the Stirling formula and the condition $\omega(t)=o\left(t^{d}\right)$, $d:=\varrho-\delta$, that $A S_{\varrho, \delta}^{m, \omega}(\Omega) \subset S_{\varrho, \delta}^{m, \omega}(\Omega)$. We observe that given two weight functions $\sigma=O(\omega)$, each amplitude of finite order with respect to $\omega$ is also an amplitude with respect to $\sigma$, and thus the corresponding pseudodifferential operator admits a continuous linear extension to $\mathcal{D}_{(\sigma)}(\Omega)$ which takes values in $\mathcal{E}_{(\sigma)}(\Omega)$.

Now we give examples of amplitudes and of pseudodifferential operators.

EXAMPLE 2.11. (1) $\omega(t):=\log (1+t)$. This is a limit case that we are not considering, since $\omega$ does not have property $(\gamma)$ in 1.1. Then $\mathcal{E}_{(\omega)}(\Omega)=$ $C^{\infty}(\Omega)$ and $\varphi^{*}(t)=\infty$ for every $t>1$. It follows that $a(x, y, \xi)$ is an amplitude of $(\omega)$-class in $S_{\varrho, \delta}^{m, \omega}(\Omega)$ according to Definition 1.6 if, and only if, for every compact set $K \subset \Omega$ and every $\alpha, \beta, \gamma \in \mathbb{N}_{0}^{p}$ there is a constant $C>0$ such that

$$
\left|D_{x}^{\alpha} D_{y}^{\gamma} D_{\xi}^{\beta} a(x, y, \xi)\right| \leq C(1+|\xi|)^{m+\delta(|\alpha+\gamma|)-\varrho|\beta|}
$$

for $x, y \in K$. This means that $a(x, y, \xi)$ is a symbol in the sense of Grigis and Sjöstrand [13].

(2) $\omega(t):=t^{d}, 0<d<1$. Then $\mathcal{E}_{(\omega)}(\Omega)$ is a Gevrey class of Beurling type. In this case $n \varphi^{*}(t / n)=(t / d) \log (t / n d)-t / d$ and it follows from the Stirling formula that for every $n \in \mathbb{N}$ there are positive constants $A_{n}$ and $B_{n}$ such that for every $\alpha \in \mathbb{N}_{0}^{p}$,

$$
A_{n}(\alpha !)^{1 / d}\left(\frac{1}{n d}\right)^{|\alpha| / d} \leq e^{n \varphi^{*}(|\alpha| / n)} \leq B_{n}(\alpha !)^{1 / d}\left(\frac{2 p}{n d}\right)^{|\alpha| / d} .
$$

Thus, $a(x, y, \xi)$ is an amplitude in $A S_{1,0}^{m, \omega}(\Omega)$ if, and only if, for every compact set $K \subset \Omega$ there are $R, B \geq 1$ such that for every $\lambda>0$ there is $C>0$ satisfying

$$
\left|D_{x}^{\alpha} D_{y}^{\gamma} D_{\xi}^{\beta} a(x, y, \xi)\right| \leq C B^{\beta} \beta !(\alpha !)^{1 / d}(\gamma !)^{1 / d} \lambda^{|\alpha+\gamma|} e^{m \omega(\xi)}(1+|\xi|)^{-|\beta|}
$$

for every $(x, y) \in K$ and $|\xi| \geq R(\beta \lambda)^{1 / d}$. This should be compared with the definition of amplitude of Gevrey class (of Roumieu type) which can be found for instance in Rodino [23].

(3) Linear partial differential operators with coefficients in $\mathcal{E}_{(\omega)}(\Omega)$ are examples of pseudodifferential operators defined by symbols of finite order. 
(4) Let $K(x, y) \in \mathcal{E}_{(\omega)}(\Omega \times \Omega)$. The integral operator with kernel $K$, $(A \varphi)(x)=\int K(x, y) \varphi(y) d y$, is a pseudodifferential operator. In fact, given any $\chi \in \mathcal{D}_{(\omega)}\left(\mathbb{R}^{p}\right)$ with $\int \chi=1, a(x, y, \xi):=K(x, y) e^{-i(x-y) \xi} \chi(\xi)$ is an amplitude in $A S_{1,0}^{0, \omega}(\Omega)$. This can be easily deduced from the compactness of the support of $\chi$ and the fact that $K \in \mathcal{E}_{(\omega)}(\Omega \times \Omega)$.

The operators $T: \mathcal{D}_{(\omega)}(\Omega) \rightarrow \mathcal{E}_{(\omega)}(\Omega)$ admitting a continuous linear extension $\widetilde{T}: \mathcal{E}_{(\omega)}^{\prime}(\Omega) \rightarrow \mathcal{E}_{(\omega)}(\Omega)$ are called $(\omega)$-smoothing. These are exactly the integral operators defined by kernels in $\mathcal{E}_{(\omega)}(\Omega \times \Omega)$.

(5) Every ultradifferential operator in the sense of Komatsu [17, p. 42] defines a pseudodifferential operator. We recall that an ultradifferential operator of $(\omega)$-class in the sense of Komatsu is an operator $G(x, D):=$ $\sum_{\alpha \in \mathbb{N}_{0}^{p}} a_{\alpha}(x) D^{\alpha}$ with $a_{\alpha} \in \mathcal{E}_{(\omega)}(\Omega)$ satisfying the following condition: there exists $m \in \mathbb{N}$ such that for every compact set $K \subset \Omega$ and every $n \in \mathbb{N}$ there is $C_{n}>0$ with

$$
\sup _{x \in K}\left|D^{\beta} a_{\alpha}(x)\right| \leq C_{n} e^{n \varphi^{*}(|\beta| / n)} e^{-m \varphi^{*}(|\alpha| / m)}
$$

for every $\alpha, \beta \in \mathbb{N}_{0}^{p}$.

It is easy to prove that $p(x, \xi):=(2 \pi)^{-p} \sum_{\alpha \in \mathbb{N}_{0}^{p}} a_{\alpha}(x) \xi^{\alpha}$ is a symbol in $A S_{1,0}^{k, \omega}(\Omega)$ for some $k \geq m$. Moreover, for every $\varphi \in \mathcal{D}_{(\omega)}(\Omega)$ we have

$$
G(x, D) \varphi=\frac{1}{(2 \pi)^{p}} \sum_{\alpha \in \mathbb{N}_{0}^{p}} a_{\alpha}(x) \int e^{i x \xi} \xi^{\alpha} \widehat{\varphi}(\xi) d \xi=P(x, D) \varphi .
$$

Examples of ultradifferential operators in the sense of Komatsu are the partial differential operators with coefficients in the class $\mathcal{E}_{(\omega)}(\Omega)$ as well as the ultradifferential operators with constant coefficients $([7])$. In this case $G(z):=\sum_{\alpha \in \mathbb{N}_{0}^{p}} a_{\alpha} z^{\alpha}$ is an entire function satisfying $\log |G(z)|=O(\omega(z))$. Therefore an application of the Paley-Wiener theorem $([8,7.3])$ gives the existence of an element $\mu \in \mathcal{E}_{(\omega)}^{\prime}\left(\mathbb{R}^{p}\right)$ with support $\{0\}$ such that $G(D) \varphi=$ $\mu * \varphi$ for every $\varphi \in \mathcal{D}_{(\omega)}\left(\mathbb{R}^{p}\right)$.

(6) If $f \in \mathcal{E}_{(\omega)}(\Omega)$ then $a(x, y, \xi):=(2 \pi)^{-p} f(x) \in A S_{1,0}^{0, \omega}(\Omega)$. Thus the operator $\varphi \mapsto f \varphi$ is a pseudodifferential operator.

(7) Let $f \in \mathcal{D}_{(\omega)}(\mathbb{R})$ be a test function with $\operatorname{supp} f=[-1,1]$ and $f(0) \neq 0$. We put $\varphi:=f \chi_{[0, \infty[}$, which is an ultradistribution with compact support and $\operatorname{sing}_{(\omega)} \operatorname{supp} \varphi=\{0\}$. Then $\widehat{\varphi}(\xi)$ is a symbol and the associated pseudodifferential operator is the operator $\psi \mapsto 2 \pi(\varphi * \psi)$. (See the comment after Theorem 2.18.)

We first observe that for every $N \in \mathbb{N}$ we have

$$
\widehat{\varphi}(\xi)=\sum_{k=0}^{N} \frac{f^{(k)}(0)}{(i \xi)^{k+1}}+\frac{1}{(i \xi)^{N+1}} \int_{0}^{1} f^{(N+1)}(t) e^{-i t \xi} d t .
$$


This follows after integrating by parts $N$ times in $\widehat{\varphi}(\xi)=\int_{0}^{1} f(t) e^{-i t \xi} d t$, using the fact that $f$ and all its derivatives vanish at $t=1$.

Hence

$$
\begin{aligned}
\widehat{\varphi}^{(N)}(\xi) & =\sum_{k=0}^{N}(-1)^{N} \frac{f^{(k)}(0)}{(i \xi)^{k+1}} N !\left(\begin{array}{c}
N+k \\
k
\end{array}\right) \frac{1}{\xi^{N}} \\
& +\frac{1}{\xi^{N} i^{N+1}} \sum_{k=0}^{N}\left(\begin{array}{c}
N \\
k
\end{array}\right) \frac{(N+k) !}{N !}(-1)^{k} \frac{1}{\xi^{k+1}} \int_{0}^{1}(-i t)^{N-k} f^{(N+1)}(t) e^{-i t \xi} d t .
\end{aligned}
$$

For every $n \in \mathbb{N}$ we put $C_{n}:=|f|_{n}$ and assume that $\log |\xi| \geq(n / N) \varphi^{*}(N / n)$. Then for every $k \leq N$ we have $\left|f^{(k)}(0)\right| /|\xi|^{k} \leq C_{n}$ and

$$
\left|\sum_{k=0}^{N}(-1)^{N} \frac{f^{(k)}(0)}{(i \xi)^{k+1}} N !\left(\begin{array}{c}
N+k \\
k
\end{array}\right) \frac{1}{\xi^{N}}\right| \leq \frac{C_{n} 4^{N} N !}{|\xi|^{N+1}} .
$$

Moreover, since $|\xi|^{k+1} \geq e^{n \varphi^{*}(k / n)} \geq \varepsilon k$ ! for some $\varepsilon>0$, there is $D_{n}>0$ with

$$
\begin{array}{r}
\left|\sum_{k=0}^{N}\left(\begin{array}{c}
N \\
k
\end{array}\right) \frac{(N+k) !}{N !}(-1)^{k} \frac{1}{\xi^{k+1}} \int_{0}^{1}(-i t)^{N-k} f^{(N+1)}(t) e^{-i t \xi} d t\right| \\
\leq D_{n} 8^{N} e^{n \varphi^{*}((N+1) / n)} .
\end{array}
$$

We conclude that

$$
\left|\widehat{\varphi}^{(N)}(\xi)\right| \leq A_{n} B^{N}|\xi|^{-N} e^{n \varphi^{*}(N / n)}
$$

for some $A_{n}, B>0$ and for every $\xi$ with $\log |\xi| \geq(n / N) \varphi^{*}(N / n)$.

Our next example is less obvious. It shows that the class of operators under consideration contains not only ultradifferential operators but also parametrices under some extra assumptions.

Proposition 2.12. Let $\omega$ be a weight with $\omega(t)=o\left(t^{d}\right), d<1$, and let $G(D)$ be an $(\omega)$-ultradifferential operator with constant coefficients such that $G(\xi)$ does not vanish on $\mathbb{R}^{p}$. If one of the following two conditions is satisfied:

(1) $G(D)$ is elliptic, or

(2) $G(D): \mathcal{E}_{(\omega)}\left(\mathbb{R}^{p}\right) \rightarrow \mathcal{E}_{(\omega)}\left(\mathbb{R}^{p}\right)$ is surjective and $\left\{t^{d}\right\}$-hypoelliptic,

then there exists a pseudodifferential operator of $(\omega)$-class $P: \mathcal{D}_{(\omega)}\left(\mathbb{R}^{p}\right) \rightarrow$ $\mathcal{E}_{(\omega)}\left(\mathbb{R}^{p}\right)$ such that $G(D) \circ P$ is the identity on $\mathcal{D}_{(\omega)}\left(\mathbb{R}^{p}\right)$.

Proof. (1) We know from [11, Thms. 3, 4] and [3, 2.1] that there exists a constant $A>0$ such that the entire function $G$ has no zeros in $\left\{z \in \mathbb{C}^{p}\right.$ : $|\operatorname{Im} z| \leq A|\operatorname{Re} z|\}$ and

$$
|G(\xi)| \geq A e^{-(1 / A) \omega(\xi)}, \quad \xi \in \mathbb{R}^{p} .
$$


Applying the minimum-modulus theorem of Chou [10, II.2.1] as in [3, 2.6, 2.8], we may find a new constant $C>0$ such that

$$
|G(z)|^{-1} \leq C e^{C \omega(z)}
$$

for $z \in \mathbb{C}^{p}$ with $|\operatorname{Im} z| \leq|\operatorname{Re} z| / C$. Since $1 / G$ is holomorphic in $\{z \in$ $\left.\mathbb{C}^{p}:|\operatorname{Im} z|<|\operatorname{Re} z| / C\right\}$, we conclude from the Cauchy inequalities that $\left((2 \pi)^{p} G\right)^{-1} \in A S_{1,0}^{m, \omega}\left(\mathbb{R}^{p}\right)$ for some $m$. The pseudodifferential operator $P$ defined by this symbol is the convolution operator defined by a fundamental solution of $G(D)$, hence $G(D) \circ P \varphi=\varphi$ for every $\varphi \in \mathcal{D}_{(\omega)}\left(\mathbb{R}^{p}\right)$.

To prove (2), we may proceed as before taking into account that there is a constant $A>0$ such that $G(\xi) \neq 0$ whenever $|\operatorname{Im} z| \leq A|\operatorname{Re} z|^{d}[3,3.3]$. In this case, the operator $P$ is defined by a symbol in $A S_{d, 0}^{m, \omega}\left(\mathbb{R}^{p}\right)$.

We recall that a continuous linear operator $T: \mathcal{D}_{(\omega)}(\Omega) \rightarrow \mathcal{E}_{(\omega)}(\Omega)$ is properly supported if the support of its kernel is a proper set in $\Omega \times \Omega$. Just as for $C^{\infty}$, this implies that $T$ can be extended as a continuous linear map from $\mathcal{E}_{(\omega)}(\Omega)$ to $\mathcal{E}_{(\omega)}(\Omega)$.

REMARK 2.13. Observe that the solution operator $P$ in 2.12 does not admit a continuous linear extension $\widetilde{P}: \mathcal{E}_{(\omega)}\left(\mathbb{R}^{p}\right) \rightarrow \mathcal{D}_{(\omega)}^{\prime}\left(\mathbb{R}^{p}\right)$ ([4, Prop. 8]), therefore it is not properly supported.

We already know that partial differential operators with coefficients in $\mathcal{E}_{(\omega)}(\Omega)$ and ultradifferential operators are examples of pseudodifferential operators. Next we see that in most cases pseudodifferential operators of $(\omega)$-class can be expressed as the composition of an ultradifferential operator of $(\omega)$-class and a finite order pseudodifferential operator. The argument depends on the possibility of constructing ultradifferential operators on Beurling spaces which are elliptic in a very strong sense. See for instance $[7,19]$.

Proposition 2.14. Let $P(x, D)$ be the pseudodifferential operator associated to $p(x, \xi) \in A S_{\varrho, \delta}^{m, \omega}(\Omega)$. Then there is an ultradifferential operator $G(D)$ of $(\omega)$-class and a symbol $q(x, \xi) \in A S_{\varrho, \delta}^{m, \omega}(\Omega)$ of finite order such that if $Q(x, D)$ is the corresponding pseudodifferential operator, then $P(x, D)=Q(x, D) \circ G(D)$.

Proof. We take $D>0$ such that $D \omega(\xi / 2)>m \omega(\xi)$. Let $G$ be an even entire function satisfying $\log |G(z)|=O(\omega(z))$ as $|z| \rightarrow \infty$ and $|G(z)| \geq$ $e^{D \omega(z)}$ whenever $|\operatorname{Im} z| \leq|\operatorname{Re} z| / D$ (the existence of such a function follows from [19, Corollary 1.4]). Then $1 / G$ is a symbol as in 2.10. Indeed, it is clear that $|1 / G(\xi)| \leq e^{-D \omega(\xi)}$ for $\xi \in \mathbb{R}^{p}$, and since it is holomorphic in $\left\{z \in \mathbb{C}^{p}:|\operatorname{Im} z|<|\operatorname{Re} z| / D\right\}$, we conclude from the Cauchy integral formula 
that, for some $C>0$ and $\xi$ large enough,

$$
\left|\left(\frac{1}{G(\xi)}\right)^{(\beta)}\right| \leq C^{|\beta|} \beta ! \frac{e^{-D \omega(\xi / 2)}}{|\xi|^{|\beta|}} \leq C^{|\beta|} \beta ! \frac{e^{-D \omega(\xi / 2)}}{|\xi| \varrho|\beta|} .
$$

We define $q(x, \xi)=p(x, \xi) / G(\xi)$. It is easy to see that $q$ is a symbol of finite order. Moreover, for every $\varphi \in \mathcal{D}_{(\omega)}(\Omega)$ we have

$$
\begin{aligned}
(Q(x, D) \circ G(D))(\varphi) & =\int q(x, \xi) e^{i x \xi} \widehat{G(D)(\varphi)}(\xi) d \xi \\
& =\int q(x, \xi) e^{i x \xi} G(\xi) \widehat{\varphi}(\xi) d \xi=P(x, D)(\varphi) .
\end{aligned}
$$

REMARK 2.15. The ultradifferential operator $G(D): \mathcal{D}_{(\omega)}^{\prime}(\Omega) \rightarrow \mathcal{D}_{(\omega)}^{\prime}(\Omega)$ in the proposition above has the property that $G(D) f \in \mathcal{E}_{(\omega)}(\Omega)$ if, and only if, $f \in \mathcal{E}_{(\omega)}(\Omega)[3,2.1]$. Hence the decomposition given in Proposition 2.14 could be useful in order to study hypoellipticity.

We observe that each ultradifferential operator of $(\omega)$-class acts continuously from $\mathcal{D}_{(\sigma)}(\Omega)$ into $\mathcal{E}_{(\sigma)}(\Omega)$ for any weight $\sigma \geq \omega$, whereas each pseudodifferential operator of $(\omega)$-class and finite order is also a pseudodifferential operator of $(\tau)$-class for $\tau \leq \omega$. However, one cannot expect that pseudodifferential operators of infinite order of $(\omega)$-class be pseudodifferential operators of a different class. Roughly speaking, the p.d.o. (of infinite order) of $(\omega)$-class are strongly tied to the Beurling space $\mathcal{D}_{(\omega)}(\Omega)$.

Next, we analyze the behaviour of a pseudodifferential operator when it is defined by an amplitude which does not depend on the $x$-variable. A combination of the next result and Proposition 2.4 will be useful to study the composition of p.d.o.'s in Theorem 3.18.

Proposition 2.16. Let $b(y, \xi)$ be an amplitude in $S_{\varrho, \delta}^{m, \omega}(\Omega)$, and let $B$ be the associated pseudodifferential operator. Then $B f \in \mathcal{D}_{L_{1},(\omega)}\left(\mathbb{R}^{p}\right)$ for every $f \in \mathcal{D}_{(\omega)}(\Omega)$, and $B: \mathcal{D}_{(\omega)}(\Omega) \rightarrow \mathcal{D}_{L_{1},(\omega)}\left(\mathbb{R}^{p}\right)$ is continuous.

Proof. Clearly $(B f)(x)=\int\left(\int b(y, \xi) e^{i(x-y) \xi} f(y) d y\right) d \xi$ can be defined for each $x \in \mathbb{R}^{p}$, and $B: \mathcal{D}_{(\omega)}(\Omega) \rightarrow \mathcal{E}_{(\omega)}\left(\mathbb{R}^{p}\right)$ is linear and continuous.

To show that $B f \in \mathcal{D}_{L_{1},(\omega)}\left(\mathbb{R}^{p}\right)$ it is enough to check that $B f \in L_{1}$ and $G(D)(B f)$ is in $L_{1}$ for each ultradifferential operator $G(D)$ of $(\omega)$-class ([1, $2.11])$.

We define $I(\xi)=\int b(y, \xi) f(y) e^{-i y \xi} d y$, so that $(B f)(x)=\int I(\xi) e^{i x \xi} d \xi$. Then for each $\alpha \in \mathbb{N}_{0}^{p}$,

$$
D_{\xi}^{\alpha} I(\xi)=\int f(y) D_{\xi}^{\alpha}\left(b(y, \xi) e^{-i y \xi}\right) d y,
$$

hence, using the Leibniz formula and 1.9 for the function $y^{\beta} f(y), \beta \leq \alpha$, we find, for every $k>0$ and each multi-index $\alpha$, a constant $C_{\alpha, k}$ such that

$$
\left|D_{\xi}^{\alpha} I(\xi)\right| \leq C_{\alpha, k} e^{-k \omega(\xi)}
$$


Therefore $D_{\xi}^{\alpha} I \in L_{1}$ and

$$
x^{\alpha}(B f)(x)=\int I(\xi) D_{\xi}^{\alpha}\left(e^{i x \xi}\right) d \xi=(-1)^{|\alpha|} \int D_{\xi}^{\alpha} I(\xi) e^{i x \xi} d \xi .
$$

Consequently, $x^{\alpha}(B f)$ is bounded for each $\alpha$, thus $B f$ is integrable. Moreover, from $1.9, D_{x}^{\alpha}\left(e^{i x \xi} I(\xi)\right)=\xi^{\alpha} I(\xi) e^{i x \xi} \in L_{1}$, and hence $D_{x}^{\alpha}(B f)(x)=$ $\int \xi^{\alpha} I(\xi) e^{i x \xi} d \xi$

Let $G(D)=\sum_{\alpha} a_{\alpha} D^{\alpha}$ be an ultradifferential operator of $(\omega)$-class. There exist $m \in \mathbb{N}$ and $C>0$ such that $\left|a_{\alpha}\right| \leq C e^{-m \varphi^{*}(|\alpha| / m)}$. From the estimates above and Lemma 1.4 we conclude that

$$
G(D)(B f)(x)=\int\left(\int b(y, \xi) G(\xi) e^{i(x-y) \xi} f(y) d y\right) d \xi .
$$

Since $G(z)$ is an entire function and $\log |G(z)|=O(\omega(z))$ we infer that $b(y, \xi) G(\xi)$ is an amplitude in some $S_{\varrho, \delta}^{k, \omega}(\Omega)$. Proceeding as before, we have $G(D)(B f) \in L_{1}$. The continuity of $B: \mathcal{D}_{(\omega)}(\Omega) \rightarrow \mathcal{D}_{L_{1},(\omega)}\left(\mathbb{R}^{p}\right)$ follows from the closed graph theorem.

We close this section by showing that pseudodifferential operators are pseudolocal, that is, they shrink singular supports. We recall that the $(\omega)$ singular support of an ultradistribution $T \in \mathcal{D}_{(\omega)}^{\prime}(\Omega)$ is the complement of the largest open subset $U$ with the property that $T \in \mathcal{E}_{(\omega)}(U)$.

Let $a(x, y, \xi)$ be an amplitude in $S_{\varrho, \delta}^{m, \omega}(\Omega)$ with associated pseudodifferential operator $A: \mathcal{D}_{(\omega)}(\Omega) \rightarrow \mathcal{E}_{(\omega)}(\Omega)$ and let $K \in \mathcal{D}_{(\omega)}^{\prime}(\Omega \times \Omega)$ be the kernel of $A$. We consider a test function $\Psi \in \mathcal{D}_{(\omega)}\left(\mathbb{R}^{p}\right)$ such that $\Psi(\xi)=1$ for $|\xi| \leq 1$ and $\Psi(\xi)=0$ for $|\xi| \geq 2$ and we put $K_{n}(x, y):=\int a(x, y, \xi) e^{i(x-y) \xi} \Psi\left(\xi / 2^{n}\right) d \xi$. It follows from Lemma 2.1 and Theorem 2.2 that $K_{n} \in \mathcal{E}_{(\omega)}(\Omega \times \Omega)$ and $K=\mathcal{D}_{(\omega)}^{\prime}(\Omega \times \Omega)-\lim _{n \rightarrow \infty} K_{n}$.

THEOREM 2.17. The $(\omega)$-singular support of the kernel $K$ of a pseudodifferential operator $A$ is contained in $\Delta:=\{(x, y) \in \Omega \times \Omega: x=y\}$.

Proof. Given $\left(x_{0}, y_{0}\right) \in(\Omega \times \Omega) \backslash \Delta$ we take a relatively compact open neighbourhood $Q$ of $\left(x_{0}, y_{0}\right)$, disjoint from $\Delta$. We show that $\left(K_{n}\right)$ is a Cauchy sequence in $\mathcal{E}_{(\omega)}(Q)$. Without loss of generality we may take $1 \leq l \leq p$ and $c_{0}>0$ such that $\left|x_{l}-y_{l}\right| \geq c_{0}$ for every $(x, y) \in Q$. Let $R \geq 3 / c_{0}$ and let $\left(C_{k}\right)$ be a sequence of positive constants such that

$$
\begin{aligned}
& \left|D_{x}^{\alpha} D_{y}^{\gamma} D_{\xi}^{\beta} a(x, y, \xi)\right| \\
& \quad \leq C_{k} e^{(\varrho-\delta) k \varphi^{*}(|\alpha+\gamma| / k)+(\varrho-\delta) k \varphi^{*}(|\beta| / k)}(1+|\xi|)^{|\alpha+\gamma| \delta-|\beta| \varrho} e^{m \omega(\xi)}
\end{aligned}
$$

for every $k \in \mathbb{N},(x, y) \in Q$ and $\xi$ with $\log (|\xi| / R) \geq k /|\beta| \varphi^{*}(|\beta| / k)$.

We fix $\bar{k} \in \mathbb{N}$, and we take $k>\bar{k}$ to be chosen later. For every $N \in \mathbb{N}$ we have, after integrating by parts $N$ times, 


$$
\begin{aligned}
D_{x}^{\alpha} D_{y}^{\gamma}\left(K_{n}(x, y)\right. & \left.-K_{n+1}(x, y)\right) \\
= & \sum_{\beta \leq \alpha} \sum_{\mu \leq \gamma}\left(\begin{array}{l}
\alpha \\
\beta
\end{array}\right)\left(\begin{array}{l}
\gamma \\
\mu
\end{array}\right) \frac{(-1)^{N+\mu}}{\left(x_{l}-y_{l}\right)^{N}} \int e^{i(x-y) \xi} \lambda_{N, \alpha, \beta, \gamma, \mu} d \xi,
\end{aligned}
$$

where

$$
\begin{aligned}
& \lambda_{N, \alpha, \beta, \gamma, \mu}=D_{\xi l}^{N}\left\{\xi^{\mu+\beta} D_{x}^{\alpha-\beta} D_{y}^{\gamma-\mu} a(x, y, \xi)\left(\Psi\left(\frac{\xi}{2^{n}}\right)-\Psi\left(\frac{\xi}{2^{n+1}}\right)\right)\right\} \\
& =\sum \frac{N !}{N_{1} ! N_{2} ! N_{3} !} \frac{\left(\mu_{l}+\beta_{l}\right) !}{\left(\mu_{l}+\beta_{l}-N_{1}\right) !} \xi^{\mu+\beta-N_{1} e_{l}} D_{\xi_{l}}^{N_{2}}\left(\Psi\left(\frac{\xi}{2^{n}}\right)-\Psi\left(\frac{\xi}{2^{n+1}}\right)\right) \\
& \quad \times D_{\xi_{l}}^{N_{3}} D_{x}^{\alpha-\beta} D_{y}^{\gamma-\mu} a(x, y, \xi),
\end{aligned}
$$

and the last sum extends over all $N_{1}, N_{2}, N_{3}$ such that $N_{1}+N_{2}+N_{3}=N$ and $N_{1} \leq \mu_{l}+\beta_{l}$. Here $e_{l}$ is the multi-index with 1 in the $l$ th position and 0 elsewhere. The support of $\Psi\left(\xi / 2^{n}\right)-\Psi\left(\xi / 2^{n+1}\right)$ is contained in $2^{n} \leq$ $|\xi| \leq 2^{n+2}$ and this inequality implies that $\left|\xi^{\mu+\beta-N_{1} e_{l}}\right| \leq|\xi|^{|\mu+\beta|-N_{1}} \leq$ $\left(2^{n+2}\right)^{|\mu+\beta|} /\left(2^{n}\right)^{N_{1}}$. We also have

$$
\left|D_{\xi_{l}}^{N_{2}}\left(\Psi\left(\frac{\xi}{2^{n}}\right)-\Psi\left(\frac{\xi}{2^{n+1}}\right)\right)\right| \leq 2|\Psi|_{k} e^{k \varphi^{*}\left(N_{2} / k\right)} \frac{1}{\left(2^{n}\right)^{N_{2}}} .
$$

Let $N \in \mathbb{N}$ be such that $(k / N) \varphi^{*}(N / k) \leq \log \left(2^{n} / R^{1 /(\varrho-\delta)}\right)$. Then $\log (|\xi| / R)$ $\geq k / N_{3} \varphi^{*}\left(N_{3} / k\right)$ and consequently, using the fact that $\varphi^{*}(t) / t$ is increasing and Lemma 1.4(1), we have

$$
\begin{aligned}
& \left|D_{x}^{\alpha-\beta} D_{y}^{\gamma-\mu} D_{\xi_{l}}^{N_{3}} a(x, y, \xi)\right| \\
& \quad \leq C_{k} e^{(\varrho-\delta) k \varphi^{*}(|\alpha-\beta+\gamma-\mu| / k)+(\varrho-\delta) k \varphi^{*}\left(N_{3} / k\right)}(1+|\xi|)^{|\alpha-\beta+\gamma-\mu| \delta-N_{3} \varrho} e^{m \omega(\xi)} \\
& \quad \leq C_{k} e^{\bar{k} \varphi^{*}(|\alpha-\beta+\gamma-\mu| / \bar{k})}\left(\frac{e^{k \varphi^{*}\left(N_{3} / k\right)}}{\left(2^{n}\right)^{N_{3}}}\right)^{\varrho-\delta} e^{(m+\bar{k}) \omega\left(2^{n+3}\right)} .
\end{aligned}
$$

Since $N_{1} ! \leq E_{k} e^{k \varphi^{*}\left(N_{1} / k\right)}$, we deduce

$$
\begin{aligned}
\left|\lambda_{N, \alpha, \beta, \gamma, \mu}\right| \leq & \sum \frac{N !}{N_{1} ! N_{2} ! N_{3} !} \frac{\left(\mu_{l}+\beta_{l}\right) !}{\left(\mu_{l}+\beta_{l}-N_{1}\right) ! N_{1} !} \\
& \times 2 C_{k} E_{k}|\Psi|_{k} \frac{e^{k \varphi^{*}\left(N_{1} / k\right)}}{\left(2^{n}\right)^{N_{1}}} \frac{e^{k \varphi^{*}\left(N_{2} / k\right)}}{\left(2^{n}\right)^{N_{2}}}\left(\frac{e^{k \varphi^{*}\left(N_{3} / k\right)}}{\left(2^{n}\right)^{N_{3}}}\right)^{\varrho-\delta} \\
& \times e^{\bar{k} \varphi^{*}(|\alpha-\beta+\gamma-\mu| / \bar{k})}\left(2^{n+2}\right)^{|\mu+\beta|} e^{(m+\bar{k}) \omega\left(2^{n+3}\right)} .
\end{aligned}
$$

Since

$$
\log \left(\frac{2^{n}}{R}\right) \geq \frac{k}{N} \varphi^{*}\left(\frac{N}{k}\right) \geq \frac{k}{N_{i}} \varphi^{*}\left(\frac{N_{i}}{k}\right) \quad(i=1,2)
$$


we have $e^{k \varphi^{*}\left(N_{i} / k\right)} /\left(2^{n}\right)^{N_{i}} \leq 1$. On the other hand,

$$
\begin{gathered}
\varphi^{*}\left(\frac{N_{1}}{k}\right)+\varphi^{*}\left(\frac{N_{2}}{k}\right)+\varphi^{*}\left(\frac{N_{3}}{k}\right) \leq \varphi^{*}\left(\frac{N}{k}\right), \\
\sum_{N_{1}+N_{2}+N_{3}=N} \frac{N !}{N_{1} ! N_{2} ! N_{3} !}=3^{N}, \quad \sum_{N_{1} \leq \mu_{l}+\beta_{l}} \frac{\left(\mu_{l}+\beta_{l}\right) !}{\left(\mu_{l}+\beta_{l}-N_{1}\right) ! N_{1} !} \leq 2^{|\mu+\beta|}
\end{gathered}
$$

and $\left(2^{n+3}\right)^{|\mu+\beta|} \leq e^{\bar{k} \omega\left(2^{n+3}\right)+\bar{k} \varphi^{*}(|\mu+\beta| / \bar{k})}$. Hence

$$
\begin{aligned}
\left|\lambda_{N, \alpha, \beta, \gamma, \mu}\right| & \leq 2 C_{k} E_{k}|\Psi|_{k} 3^{N}\left(\frac{e^{k \varphi^{*}(N / k)}}{\left(2^{n}\right)^{N}}\right)^{\varrho-\delta} e^{\bar{k} \varphi^{*}(|\alpha+\gamma| / \bar{k})} e^{(m+2 \bar{k}) \omega\left(2^{n+3}\right)} \\
& :=I_{N, \alpha, \gamma} .
\end{aligned}
$$

Since the support of $\lambda_{N, \alpha, \beta, \gamma, \mu}(x, y, \cdot)$ is contained in the set $2^{n} \leq|\xi| \leq 2^{n+2}$, which has measure $\left(2^{n+1}\right)^{p}\left(4^{p}-1\right)$, we finally obtain

$$
\left|D_{x}^{\alpha} D_{y}^{\gamma}\left(K_{n}(x, y)-K_{n+1}(x, y)\right)\right| \leq 2^{|\alpha+\gamma|}\left(2^{n+1}\right)^{p}\left(4^{p}-1\right) \frac{1}{c_{0}^{N}} I_{N, \alpha, \gamma} .
$$

We put $R^{*}:=R^{1 /(\varrho-\delta)}$. Then, since $3 / c_{0} \leq R$, we deduce that

$$
\begin{aligned}
q_{Q, \bar{k}}\left(K_{n}-K_{n+1}\right) \leq & 2 E_{k} C_{k}|\Psi|_{k}\left(2^{n+1}\right)^{p}\left(4^{p}-1\right) \\
& \times\left(\frac{\left(R^{*}\right)^{N} e^{k \varphi^{*}(N / k)}}{\left(2^{n}\right)^{N}}\right)^{\varrho-\delta} e^{(m+2 \bar{k}) \omega\left(2^{n+3}\right)}
\end{aligned}
$$

whenever $\log \left(2^{n} / R^{*}\right) \geq(k / N) \varphi^{*}(N / k)$. Observe that the estimates just obtained also hold if we replace $N$ by $j \leq N$. Now, if we select $k$ large enough, and take $N$ such that

$$
\frac{k}{N} \varphi^{*}\left(\frac{N}{k}\right) \leq \log \left(\frac{2^{n}}{R^{*}}\right)<\frac{k}{N+1} \varphi^{*}\left(\frac{N+1}{k}\right)
$$

an application of Lemma 1.5 gives

$$
q_{Q, \bar{k}}\left(K_{n}-K_{n+1}\right) \leq 2 C_{k} E_{k}|\Psi|_{k}\left(2^{n+1}\right)^{p}\left(4^{p}-1\right) e^{-\omega\left(2^{n}\right)},
$$

whence it easily follows that $\left(K_{n}\right)$ is a Cauchy sequence in $\mathcal{E}_{(\omega)}(Q)$. Thus $\left(x_{0}, y_{0}\right)$ does not belong to the $(\omega)$-singular support of $K$.

As in [25] we can conclude that the pseudodifferential operators are pseudolocal.

ThEOREM 2.18. Let $A: \mathcal{E}_{(\omega)}^{\prime}(\Omega) \rightarrow \mathcal{D}_{(\omega)}^{\prime}(\Omega)$ be the pseudodifferential operator associated to an amplitude $a(x, y, \xi)$ in $S_{\varrho, \delta}^{m, \omega}(\Omega)$. Then

$$
\operatorname{sing}_{(\omega)} \operatorname{supp}(A \mu) \subset \operatorname{sing}_{(\omega)} \operatorname{supp}(\mu)
$$

for every $\mu \in \mathcal{E}_{(\omega)}^{\prime}(\Omega)$.

If a convolution operator $\psi \mapsto \psi * S, \psi \in \mathcal{D}_{(\omega)}\left(\mathbb{R}^{p}\right), S \in \mathcal{D}_{(\omega)}^{\prime}\left(\mathbb{R}^{p}\right)$, is a pseudodifferential operator, by 2.18 the $(\omega)$-singular support of $S$ reduces 
to $\{0\}$. Therefore, not every convolution operator is a pseudodifferential operator.

3. Symbolic calculus. One of the problems one has to face is how to determine the class of symbols in order that the theory for the operators can be converted into an algebraic theory for the corresponding symbols. Moreover, the class of operators should be closed under products. This leads to the necessity of developing the classical symbolic calculus in our setting. The definitions below are motivated by [23, 25].

Definition 3.1. We denote by $F A S_{\varrho, \delta}^{m, \omega}(\Omega)$ the set of all formal sums

$$
\sum_{j \in \mathbb{N}_{0}} a_{j}(x, y, \xi)
$$

such that $a_{j}(x, y, \xi) \in C^{\infty}\left(\Omega \times \Omega \times \mathbb{R}^{p}\right)$ and for every compact set $Q \subset \Omega \times \Omega$ there are $R, B \geq 1$ and a sequence $C_{n}>0, n \in \mathbb{N}$, with

$$
\begin{aligned}
& \left|D_{x}^{\alpha} D_{y}^{\gamma} D_{\xi}^{\beta} a_{j}(x, y, \xi)\right| \\
& \quad \leq C_{n} B^{|\beta|} \beta ! e^{(\varrho-\delta) n \varphi^{*}(|\alpha+\gamma|+j / n)} e^{m \omega(\xi)}(1+|\xi|)^{|\alpha+\gamma| \delta-|\beta| \varrho-(\varrho-\delta) j}
\end{aligned}
$$

for every $j \in \mathbb{N}_{0},(x, y) \in Q$, and $\xi$ with $\log (|\xi| / R) \geq \frac{n}{|\beta|+j} \varphi^{*}\left(\frac{|\beta|+j}{n}\right)$.

Let $a \in A S_{\varrho, \delta}^{m, \omega}(\Omega)$ and put $a_{0}:=a, a_{j}:=0$ for $j \neq 0$. Then we can identify $a$ with the formal $\operatorname{sum} \sum a_{j}$.

EXAmPle 3.2. Let $a \in A S_{\varrho, \delta}^{m, \omega}(\Omega)$. Then the series $\sum_{j=0}^{\infty} p_{j}(x, \xi)$, where $p_{j}(x, \xi):=\sum_{|\alpha|=j}(1 / \alpha !) D_{\xi}^{\alpha} \partial_{y}^{\alpha} a(x, y, \xi)_{\mid y=x}$, is a formal sum in $F A S_{\varrho, \delta}^{m, \omega}(\Omega)$.

Definition 3.3. Two formal sums $\sum a_{j}$ and $\sum b_{j}$ in $F A S_{\varrho, \delta}^{m, \omega}(\Omega)$ are said to be equivalent if for every compact set $Q \subset \Omega \times \Omega$ there are $R, B \geq 1$ and two sequences $C_{n}>0$ and $N_{n}(n \in \mathbb{N})$ with

$$
\begin{aligned}
& \left|D_{x}^{\alpha} D_{y}^{\gamma} D_{\xi}^{\beta} \sum_{j<N}\left(a_{j}-b_{j}\right)\right| \\
& \quad \leq C_{n} B^{|\beta|} \beta ! e^{(\varrho-\delta) n \varphi^{*}(|\alpha+\gamma|+N / n)} e^{m \omega(\xi)}(1+|\xi|)^{|\alpha+\gamma| \delta-|\beta| \varrho-(\varrho-\delta) N}
\end{aligned}
$$

for every $(x, y) \in Q, N \geq N_{n}$, and $\xi$ with $\log (|\xi| / R) \geq \frac{n}{|\beta|+N} \varphi^{*}\left(\frac{|\beta|+N}{n}\right)$.

REMARK 3.4. If $a(x, y, \xi)=0$ for every $x, y \in \Omega$ and $|\xi| \geq M$ then $a \sim 0$. In particular, every $(\omega)$-smoothing operator is associated to an amplitude equivalent to zero.

Proposition 3.5. Let $A$ be the pseudodifferential operator defined by an amplitude $a \in A S_{\varrho, \delta}^{m, \omega}(\Omega)$ which is equivalent to zero. Then $A$ is an $(\omega)$-smoothing operator. 
Proof. We show that $K(x, y):=\int e^{i(x-y) \xi} a(x, y, \xi) d \xi$ is a function in $\mathcal{E}_{(\omega)}(\Omega \times \Omega)$ and $(A \varphi)(x)=\int K(x, y) \varphi(y) d y$ for every $\varphi \in \mathcal{D}_{(\omega)}(\Omega)$. We fix a compact set $Q \subset \Omega \times \Omega$. Then

$$
\left|D_{x}^{\alpha} D_{y}^{\gamma} a(x, y, \xi)\right| \leq C_{n} e^{(\varrho-\delta) n \varphi^{*}(|\alpha+\gamma|+N / n)} e^{m \omega(\xi)}|\xi|^{\delta|\alpha+\gamma|-(\varrho-\delta) N}
$$

for every $(x, y) \in Q, N \geq N_{n}$, and $\xi$ with $\log (|\xi| / R) \geq(n / N) \varphi^{*}(N / n)$. We now fix $n_{0} \in \mathbb{N}$ and we take $0<\varepsilon<1$ and $n \in \mathbb{N}$ with $\omega(t / R) \geq \varepsilon \omega(t)-1 / \varepsilon$ and $\varepsilon(\varrho-\delta) n>2 n_{0}$. Then, for every $N \geq N_{8 n}$ and $\xi$ with

$$
\frac{2 n}{N} \varphi^{*}\left(\frac{N}{2 n}\right) \leq \log \left(\frac{|\xi|}{R}\right) \leq \frac{2 n}{N+1} \varphi^{*}\left(\frac{N+1}{2 n}\right)
$$

we have

$$
\begin{aligned}
\left|D_{x}^{\alpha} D_{y}^{\gamma}\left(e^{i(x-y) \xi} a(x, y, \xi)\right)\right| \\
\leq C_{8 n} \sum_{\substack{\beta \leq \alpha \\
\mu \leq \gamma}}\left(\begin{array}{c}
\alpha \\
\beta
\end{array}\right)\left(\begin{array}{c}
\gamma \\
\mu
\end{array}\right) e^{(\varrho-\delta) n_{0} \varphi^{*}\left(|\alpha-\beta+\gamma-\mu| / n_{0}\right)} \\
\quad \times|\xi|^{|\beta+\mu|+\delta|\alpha-\beta+\gamma-\mu|-(\varrho-\delta) N} e^{m \omega(\xi)} e^{(\varrho-\delta) 4 n \varphi^{*}(N / 4 n)} .
\end{aligned}
$$

Applying Lemma 1.4, we have

$$
|\xi|^{\delta|\alpha-\beta+\gamma-\mu|} e^{-\delta n_{0} \varphi^{*}\left(|\alpha-\beta+\gamma-\mu| / n_{0}\right)} \leq e^{n_{0} \omega(\xi)}
$$

and

$$
|\xi|^{|\beta+\mu|} \leq e^{n_{0} \varphi^{*}\left(|\beta+\mu| / n_{0}\right)} e^{n_{0} \omega(\xi)},
$$

from which we conclude that

$$
\begin{aligned}
& \quad\left|D_{x}^{\alpha} D_{y}^{\gamma}\left(e^{i(x-y) \xi} a(x, y, \xi)\right)\right| \\
& \quad \leq C_{8 n} 2^{|\alpha+\gamma|} e^{n_{0} \varphi^{*}\left(|\alpha+\gamma| / n_{0}\right)} e^{\left(m+2 n_{0}\right) \omega(\xi)} e^{(\varrho-\delta) 4 n \varphi^{*}(N / 4 n)}|\xi|^{-(\varrho-\delta) N} .
\end{aligned}
$$

An application of Lemma 1.5(2) gives

$$
\left|D_{x}^{\alpha} D_{y}^{\gamma}\left(e^{i(x-y) \xi} a(x, y, \xi)\right)\right| \leq D_{n_{0}} 2^{|\alpha+\gamma|} e^{n_{0} \varphi^{*}\left(|\alpha+\gamma| / n_{0}\right)} e^{\left(m+1-n_{0}\right) \omega(\xi)} .
$$

Selecting $n_{0}$ large enough we conclude that $K \in \mathcal{E}_{(\omega)}(\Omega \times \Omega)$. To finish, it is easy to see that $A$ coincides with the operator with kernel $K$.

LEMma 3.6 ([24, p. 241]). There is a sequence $\left(\Phi_{l}\right)_{l \geq 1}$ and constants $C, D>0$ such that $\Phi_{l} \in \mathcal{D}_{(\omega)}\left(\mathbb{R}^{p}\right),\left|\Phi_{l}(\xi)\right| \leq 1, \Phi_{l}(\xi)=1$ for $|\xi| \leq 2$, $\Phi_{l}(\xi)=0$ for $|\xi| \geq 3$ and

$$
\left|D_{\xi}^{\alpha} \Phi_{l}(\xi)\right| \leq C(D / 3)^{|\alpha|} l^{|\alpha|+1} \quad \text { whenever }|\alpha| \leq l .
$$

We now fix a positive constant $R \geq 1$ and we put

$$
\Psi_{j, n}(\xi):=1-\Phi_{j}\left(\frac{\xi}{R e^{(n / j) \varphi^{*}(j / n)}}\right) .
$$


Then $\Psi_{j, n}(\xi) \neq 0$ implies $|\xi| \geq 2 R e^{(n / j) \varphi^{*}(j / n)}$, whereas if $\xi$ is in the support of any derivative we also have $|\xi| \leq 3 R e^{(n / j) \varphi^{*}(j / n)}$. It follows that

$$
\left|D_{\xi}^{i} \Psi_{j, n}(\xi)\right| \leq C(D /|\xi|)^{|i|} j^{|i|+1}
$$

for any multi-index $i$ with $|i| \leq j$.

In order to construct an amplitude from a formal sum, the idea is the following: For a fixed $n$, as in [25] we can find a $C^{\infty}$ function $a^{n}$ satisfying the estimates in 2.10 only for this fixed $n$. Since each $a^{n}$ is obtained as a series involving the $a_{j}$ 's, we will take, for each $n$, a finite block of the series, in such a way that when we put together these blocks we obtain an amplitude which is equivalent to the formal sum. In some sense, this procedure reflects the fact that our amplitudes are "tied up" to the Fréchet spaces $\mathcal{E}_{(\omega)}(\Omega)$.

TheOREM 3.7. Let $\sum a_{j} \in F A S_{\varrho, \delta}^{m, \omega}(U)$ and let $\Omega$ be a relatively compact open subset of $U$. Then there is an amplitude $a \in A S_{\varrho, \delta}^{m, \omega}(\Omega)$ such that $a \sim \sum a_{j}$ on $\Omega$.

Proof. We put

$$
\Psi_{j, n}(\xi):=1-\Phi_{j}\left(\frac{\xi}{R e^{(n / j) \varphi^{*}(j / n)}}\right),
$$

where $R$ will be determined later. We set $R_{1}:=(2 R)^{\varrho-\delta}$ and we observe that $\Psi_{j, n}(\xi) \neq 0$ implies $e^{(\varrho-\delta) n \varphi^{*}(j / n)} \leq|\xi|^{j(\varrho-\delta)}\left(1 / R_{1}\right)^{j}$, whereas if $\xi$ is in the support of any derivative we also have $(|\xi| / 3)^{j(\varrho-\delta)}\left(1 / R_{1}\right)^{j} \leq$ $e^{(\varrho-\delta) n \varphi^{*}(j / n)}$. According to Definition 3.1, we can select $R$ large enough so that $\sum j e^{D j p} / R_{1}^{j}<\infty$ and, for some sequence $\left(C_{n}\right)$,

$$
\begin{aligned}
& \left|D_{x}^{\alpha} D_{y}^{\gamma} D_{\xi}^{\beta} a_{j}(x, y, \xi)\right| \\
& \quad \leq C_{n} B^{|\beta|} \beta ! e^{(\varrho-\delta) 2 n \varphi^{*}(|\alpha+\gamma| / 2 n)} e^{m \omega(\xi)}|\xi|^{|\alpha+\gamma| \delta-|\beta|} e^{(\varrho-\delta) 2 n \varphi^{*}(j / 2 n)}|\xi|^{-(\varrho-\delta) j}
\end{aligned}
$$

whenever $(x, y) \in \Omega$ and $\log (|\xi| / R) \geq \frac{n}{|\beta|+j} \varphi^{*}\left(\frac{|\beta|+j}{n}\right)$.

We first assume $(x, y) \in \Omega, n \in \mathbb{N}, \log (|\xi| / 3 R) \geq(n /|\beta|) \varphi^{*}(|\beta| / n)$ and $\Psi_{j, n}(\xi) \neq 0$. Then

$$
\log \left(\frac{|\xi|}{R}\right) \geq \max \left(\frac{n}{|\beta|} \varphi^{*}\left(\frac{|\beta|}{n}\right), \frac{n}{j} \varphi^{*}\left(\frac{j}{n}\right)\right) \geq \frac{2 n}{|\beta|+j} \varphi^{*}\left(\frac{|\beta|+j}{2 n}\right) .
$$

Moreover from $D_{\xi}^{i} \Psi_{j, n}(\xi) \neq 0$ we deduce that $\log (|\xi| / 3 R) \leq(n / j) \varphi^{*}(j / n)$, and consequently, $|\beta| \leq j$ since $\varphi^{*}(t) / t$ is increasing. Since $|\xi| \geq 1$ and $0 \leq \varrho \leq 1$, it follows that $|\xi|^{-|i|} \leq|\xi|^{-|i| \varrho}$ and we can estimate

$$
\begin{aligned}
& \left|D_{x}^{\alpha} D_{y}^{\gamma} D_{\xi}^{\beta}\left(a_{j}(x, y, \xi) \Psi_{j, n}(\xi)\right)\right| e^{-m \omega(\xi)} \\
& \quad \leq \sum_{i \leq \beta}\left(\begin{array}{c}
\beta \\
i
\end{array}\right)\left|D_{\xi}^{i} \Psi_{j, n}(\xi) D_{x}^{\alpha} D_{y}^{\gamma} D_{\xi}^{\beta-i} a_{j}(x, y, \xi)\right| e^{-m \omega(\xi)}
\end{aligned}
$$




$$
\begin{aligned}
\leq & C C_{n} e^{2 n \varphi^{*}(|\alpha+\gamma| / 2 n)}|\xi|^{|\alpha+\gamma| \delta-(\varrho-\delta) j} e^{n \omega(\xi)} \sum_{i \leq \beta} \frac{B^{|\beta-i|} D^{|i|} j^{|i|+1}}{i !}|\xi|^{-|i|-\varrho|\beta-i|} \\
\leq & C C_{n} B^{|\beta|} \beta ! e^{(\varrho-\delta) 2 n \varphi^{*}(|\alpha+\gamma| / 2 n)}|\xi|^{|\alpha+\gamma| \delta-|\beta| \varrho} e^{(\varrho-\delta) 2 n \varphi^{*}(j / 2 n)}|\xi|^{-(\varrho-\delta) j} \\
& \times \sum_{i \leq \beta} \frac{D^{|i|} j^{|i|+1}}{i !} \\
\leq & C C_{n} B^{|\beta|} \beta ! e^{(\varrho-\delta) 2 n \varphi^{*}(|\alpha+\gamma| / 2 n)}|\xi|^{|\alpha+\gamma| \delta-|\beta| \varrho}\left(\frac{1}{R_{1}}\right)^{j} j e^{D j p} .
\end{aligned}
$$

By induction we select a sequence $\left(j_{n}\right)$ of natural numbers such that $j_{1}:=0$, $j_{n}<j_{n+1}, \lim _{n \rightarrow \infty} j_{n} / n=\infty$ and

$$
C_{n+1} \sum_{j=j_{n+1}+1}^{\infty} \frac{j e^{D j p}}{R_{1}^{j}} \leq \frac{C_{n}}{2} \sum_{j=j_{n}+1}^{j_{n}+2} \frac{j e^{D j p}}{R_{1}^{j}} .
$$

Then

$$
\bar{D}_{n}:=C_{n} \sum_{j=j_{n}+1}^{j_{n+1}} \frac{j e^{D j p}}{R_{1}^{j}}
$$

satisfies $\bar{D}_{n+1} \leq \bar{D}_{n} / 2$. We now prove that

$$
a(x, y, \xi):=a_{0}(x, y, \xi)+\sum_{n=1}^{\infty} \sum_{j=j_{n}+1}^{j_{n+1}} \Psi_{j, n}(\xi) a_{j}(x, y, \xi)
$$

is an amplitude. Since $j_{n} \leq j$ and $\Psi_{j, n}(\xi) \neq 0$ implies

$$
\frac{n}{j_{n}} \varphi^{*}\left(\frac{j_{n}}{n}\right) \leq \frac{n}{j} \varphi^{*}\left(\frac{j}{n}\right) \leq \log \left(\frac{|\xi|}{2 R}\right)
$$

the condition $\lim _{n \rightarrow \infty}\left(n / j_{n}\right) \varphi^{*}\left(j_{n} / n\right)=\infty$ permits us to conclude that the sum defining $a$ is locally finite. Hence $a$ is a well defined $C^{\infty}$ function. Let us assume $\log (|\xi| / 3 R) \geq(n /|\beta|) \varphi^{*}(|\beta| / n)$. Then, for every $n \in \mathbb{N}$,

$$
\begin{aligned}
& \left|D_{x}^{\alpha} D_{y}^{\gamma} D_{\xi}^{\beta}\left(\sum_{k=n}^{\infty} \sum_{j=j_{k}+1}^{j_{k+1}} \Psi_{j, k}(\xi) a_{j}(x, y, \xi)\right)\right| \\
& \quad \leq C B^{|\beta|} \beta ! e^{(\varrho-\delta) n \varphi^{*}(|\alpha+\gamma| / n)}|\xi|^{|\alpha+\gamma| \delta-|\beta| \varrho} e^{m \omega(\xi)} \sum_{k=n}^{\infty} \bar{D}_{k} .
\end{aligned}
$$

Since $a_{0}+\sum_{k=1}^{n-1} \sum_{j=j_{k}+1}^{j_{k+1}} \Psi_{j, k} a_{j}$ is a finite sum of amplitudes we conclude that $a(x, y, \xi) \in A S_{\varrho, \delta}^{m, \omega}(\Omega)$.

To finish we have to show that $a \sim \sum a_{j}$ on $\Omega$. In order to do this, we assume that $(x, y) \in \Omega$ and $\log (|\xi| / 3 R) \geq \frac{n}{|\beta|+N} \varphi^{*}\left(\frac{|\beta|+N}{n}\right)$ and we estimate 
the derivatives of

$$
a-\sum_{j<N} a_{j}=\sum_{k=1}^{\infty} \sum_{j=j_{k}+1}^{j_{k+1}} \Psi_{j, k} a_{j}-\sum_{j=1}^{N-1} a_{j} .
$$

We will only consider the case $N>n j_{n}$. For every $j \in \mathbb{N}$ there is $k \in \mathbb{N}$ with $j_{k}<j \leq j_{k+1}$. Then $k<n$ implies $j \leq j_{n}(<N)$ and

$$
\log \left(\frac{|\xi|}{3 R}\right) \geq \frac{n}{N} \varphi^{*}\left(\frac{N}{n}\right) \geq \frac{1}{j_{n}} \varphi^{*}\left(j_{n}\right) \geq \frac{k}{j} \varphi^{*}\left(\frac{j}{k}\right) .
$$

On the other hand, $k \geq n$ and $N>j$ also imply

$$
\log \left(\frac{|\xi|}{3 R}\right) \geq \frac{n}{N} \varphi^{*}\left(\frac{N}{n}\right) \geq \frac{k}{j} \varphi^{*}\left(\frac{j}{k}\right)
$$

and $\Psi_{j, k}(\xi)=1$. Consequently, $a-\sum_{j<N} a_{j}$ can be expressed as a sum of functions $\Psi_{j, k} a_{j}$ with $j \geq N$ and $k \geq n$.

It follows from the above estimates that

$$
\begin{aligned}
\left|D_{x}^{\alpha} D_{y}^{\gamma} D_{\xi}^{\beta}\left(a_{j}(x, y, \xi) \Psi_{j, k}(\xi)\right)\right| e^{-m \omega(\xi)} \\
\leq C \bar{D}_{k} B^{|\beta|} \beta ! e^{(\varrho-\delta) 2 k \varphi^{*}(|\alpha+\gamma|+N / 2 k)}|\xi|^{|\alpha+\gamma| \delta-|\beta| \varrho-(\varrho-\delta) N} \\
\quad \times e^{(\varrho-\delta) k \varphi^{*}((j-N) / k)}|\xi|^{-(\varrho-\delta)(j-N)} \\
\leq C \bar{D}_{k} B^{|\beta|} \beta ! !^{(\varrho-\delta) 2 k \varphi^{*}(|\alpha+\gamma|+N / 2 k)}|\xi|^{|\alpha+\gamma| \delta-|\beta| \varrho-(\varrho-\delta) N}\left(1 / R_{1}\right)^{j-N},
\end{aligned}
$$

where the last inequality follows from the fact that $\Psi_{j, k}(\xi) \neq 0$ implies that

$$
\log \left(\frac{|\xi|}{2 R}\right) \geq \frac{k}{j} \varphi^{*}\left(\frac{j}{k}\right) \geq \frac{k}{j-N} \varphi^{*}\left(\frac{j-N}{k}\right) .
$$

Finally, we get

$$
\begin{aligned}
\left|D_{x}^{\alpha} D_{y}^{\gamma} D_{\xi}^{\beta}\left(a-\sum_{j<N} a_{j}\right)\right| \leq & \sum_{k \geq n} \sum_{j \geq N}\left|D_{x}^{\alpha} D_{y}^{\gamma} D_{\xi}^{\beta}\left(\Psi_{j, k} a_{j}\right)\right| \\
\leq & C B^{|\beta|} \beta ! e^{(\varrho-\delta) n \varphi^{*}(|\alpha+\gamma|+N / n)}|\xi|^{|\alpha+\gamma| \delta-|\beta| \varrho-(\varrho-\delta) N} \\
& \times e^{m \omega(\xi)} \sum_{k \geq n} \bar{D}_{k}\left(\sum_{j \geq N}\left(\frac{1}{R_{1}}\right)^{j-N}\right) .
\end{aligned}
$$

We observe that any other choice of the sequence $\left(j_{n}\right)$ and the constant $R$ satisfying the estimates in the proof of the previous result would give another amplitude defining the same pseudodifferential operator, modulo an $(\omega)$-smoothing one. Hence, in what follows we can assume without loss of generality that $\left(j_{n}\right)$ and $R$ are as large as necessary.

Our next aim is to give an asymptotic expansion formula. From now on, we will always assume that $\mathcal{E}_{(\omega)}(\Omega)$ contains the Gevrey class $\Gamma^{\{s\}}(\Omega)$ 
for some $s>1$. Then, for $\sigma(t):=t^{1 / s}, \mathcal{E}_{(\sigma)}(\Omega)$ is contained (and dense) in $\mathcal{E}_{(\omega)}(\Omega)($ see $[8])$.

We assume that $(n / j) \varphi^{*}(j / n) \geq n$ for every $j \geq j_{n}$. We put $\varphi_{j}:=\Psi_{j, n}$ if $j_{n}<j \leq j_{n+1}, \varphi_{0}(\xi)=1$. As in the proof of 2.2 we have the following

Lemma 3.8. Let $a \in S_{\varrho, \delta}^{m, \omega}(\Omega)$ and let $A$ be the pseudodifferential operator defined by $a$. Then, for every $u \in \mathcal{D}_{(\omega)}(\Omega)$,

$$
A(u)=\sum_{j=0}^{\infty} A_{j}(u)
$$

where $A_{j}$ is the pseudodifferential operator with amplitude $a_{j}(x, y, \xi):=$ $\left(\varphi_{j}-\varphi_{j+1}\right)(\xi) a(x, y, \xi)$.

Lemma 3.9. Let $\sum_{j=0}^{\infty} p_{j}(x, \xi)$ be a formal sum in $F A S_{o, \delta}^{m, \omega}(U), \Omega$ a relatively compact open subset of $U$, and $\left(j_{n}\right)$ as in the proof of 3.7 and satisfying the additional assumption $(n / j) \varphi^{*}(j / n) \geq \max \left(n, \log C_{n}\right)$ for $j \geq j_{n},\left(C_{n}\right)$ being the constants of Definition 3.1 relative to the closure of $\Omega$. Let

$$
p(x, \xi):=\sum_{j=0}^{\infty} \varphi_{j}(\xi) p_{j}(x, \xi),
$$

which is a symbol in $A S_{\varrho, \delta}^{m, \omega}(\Omega)$. Then the corresponding pseudodifferential operator $P(x, D)$ is the limit in $L\left(\mathcal{D}_{(\omega)}(\Omega), \mathcal{D}_{(\omega)}^{\prime}(\Omega)\right)$ of the sequence of operators $P_{N}: \mathcal{D}_{(\omega)}(\Omega) \rightarrow \mathcal{E}_{(\omega)}(\Omega)$, where each $P_{N}$ is the pseudodifferential operator with symbol $\sum_{j=0}^{N}\left(\varphi_{j}-\varphi_{j+1}\right)(\xi)\left(\sum_{l=0}^{j} p_{l}(x, \xi)\right)$.

Proof. We first observe that

$$
\sum_{j=0}^{N}\left(\varphi_{j}-\varphi_{j+1}\right)(\xi)\left(\sum_{l=0}^{j} p_{l}(x, \xi)\right)=\sum_{j=0}^{N} \varphi_{j}(\xi) p_{j}(x, \xi)-\varphi_{N+1}(\xi) \sum_{j=0}^{N} p_{j}(x, \xi) .
$$

Let $B$ be a bounded set in $\mathcal{D}_{(\omega)}(\Omega)$ and let $K$ be a compact set in $\Omega$. We will prove:

$$
\begin{aligned}
& \text { (a) } \int\left(\sum_{j=0}^{N} \varphi_{j}(\xi) p_{j}(x, \xi)\right) e^{i x \xi} \widehat{u}(\xi) d \xi \rightarrow \int\left(\sum_{j=0}^{\infty} \varphi_{j}(\xi) p_{j}(x, \xi)\right) e^{i x \xi} \widehat{u}(\xi) d \xi \\
& \text { (b) } \int \varphi_{N+1}(\xi)\left(\sum_{j=0}^{N} p_{j}(x, \xi)\right) e^{i x \xi} \widehat{u}(\xi) d \xi \rightarrow 0
\end{aligned}
$$

as $N$ goes to infinity, uniformly in $x \in K$ and $u \in B$. By the Paley-Wiener theorem $([8,3.4])$ there is $D>0$ with $|\widehat{u}(\xi)| \leq D e^{-(m+3) \omega(\xi)}$ for all $u \in B$. It follows from Definition 3.1 that there is a sequence $\left(C_{n}\right)$ with

$$
\left|p_{j}(x, \xi)\right| \leq C_{n} \frac{e^{(\varrho-\delta) n \varphi^{*}(j / n)}}{(1+|\xi|)^{(\varrho-\delta) j}} e^{m \omega(\xi)}
$$


whenever $x \in K$ and $\log (|\xi| / R) \geq(n / j) \varphi^{*}(j / n)$. Since $\varphi_{j}(\xi) \neq 0$ and $j_{n}<j \leq j_{n+1}$ imply $\log (|\xi| / 2 R) \geq(n / j) \varphi^{*}(j / n)$ we get

$$
\left|\varphi_{j}(\xi) p_{j}(x, \xi) \widehat{u}(\xi)\right| \leq \frac{C_{n}}{(2 R)^{j(\varrho-\delta)}} D e^{-3 \omega(\xi)} .
$$

We can assume

$$
e^{-\omega(\xi)} \leq \frac{1}{|\xi|} \leq \frac{1}{e^{(n / j) \varphi^{*}(j / n)}} \quad \text { for } \xi \in \operatorname{supp} \varphi_{j} .
$$

For a fixed $N$ one can find $l$ such that $j_{l}<N \leq j_{l+1}$. Then, since $(n / j) \varphi^{*}(j / n)$ $\geq \log C_{n}$ for every $n \in \mathbb{N}$ and $j_{n}<j \leq j_{n+1}$, we have

$$
\sum_{j=N+1}^{\infty} \int\left|\varphi_{j}(\xi) p_{j}(x, \xi) \widehat{u}(\xi)\right| d \xi \leq D \sum_{n=l}^{\infty} \sum_{j=j_{n}+1}^{j_{n+1}} \frac{1}{(2 R)^{j(\varrho-\delta)}} \int e^{-2 \omega(\xi)} d \xi
$$

which proves (a). To prove (b), given $N$ we take $n$ with $j_{n}+1 \leq N+1 \leq j_{n+1}$ and we note that $\varphi_{N+1}(\xi) \neq 0$ implies $\log (|\xi| / 2 R) \geq \frac{n}{N+1} \varphi^{*}\left(\frac{N+1}{n}\right)$. Then

$$
\begin{aligned}
\left|\varphi_{N+1}(\xi)\left(\sum_{j=0}^{N} p_{j}(x, \xi)\right)\right| & \leq C_{n} \sum_{j=0}^{N} \frac{e^{n(\varrho-\delta) \varphi^{*}(j / n)}}{|\xi|^{j(\varrho-\delta)}} e^{m \omega(\xi)} \\
& \leq C_{n} \sum_{j=0}^{\infty}\left(\frac{1}{2 R}\right)^{(\varrho-\delta) j} e^{m \omega(\xi)} .
\end{aligned}
$$

Hence

$$
\left|\varphi_{N+1}(\xi)\left(\sum_{j=0}^{N} p_{j}(x, \xi)\right) \widehat{u}(\xi)\right| \leq C e^{-\omega(\xi)} e^{-\frac{n}{N+1} \varphi^{*}\left(\frac{N+1}{n}\right)}
$$

which yields (b), since $j_{n}+1 \leq N+1 \leq j_{n+1}$ implies

$$
\frac{n}{N+1} \varphi^{*}\left(\frac{N+1}{n}\right) \geq n \text {. }
$$

Lemma 3.10. For every $n \in \mathbb{N}$ we have

$$
\lim _{N \rightarrow \infty} \frac{N}{e^{(n / N) \varphi^{*}(N / n)}}=0
$$

Proof. If this is not the case, we find $0<\varepsilon<1$ and an unbounded sequence $\left(N_{k}\right)$ of natural numbers such that

$$
\varepsilon^{-N_{k}} N_{k}^{N_{k}} \geq e^{n \varphi^{*}\left(N_{k} / n\right)} .
$$

An application of the Stirling formula and [12, 2.1.2] gives a contradiction.

LEMMA 3.11. Let $m \geq n$ and

$$
\frac{1}{e} e^{(m / j) \varphi^{*}(j / m)} \leq t \leq e^{(n / j) \varphi^{*}(j / n)} .
$$


Then

$$
|t|^{j+1} \geq e^{n \omega(t)} e^{2 m \varphi^{*}(j / 2 m)} e^{-j} .
$$

In particular

$$
e^{n \varphi^{*}(j / n)} \geq e^{(n-1) \omega(t)} e^{2 n \varphi^{*}(j / 2 n)} \quad \text { for } j \text { large enough. }
$$

Proof. By Lemma 1.4(2) we know that

$$
n \omega(t) \leq \log (t)+\sup _{k \in \mathbb{N}_{0}}\left\{k \log (t)-n \varphi^{*}(k / n)\right\}
$$

for $t \geq 1$. Since $0<t \leq e^{(n / j) \varphi^{*}(j / n)}$ and $\varphi^{*}(t) / t$ is an increasing function we deduce that $n \omega(t) \leq \log (t)+l \log (t)-n \varphi^{*}(l / n)$ for some $0 \leq l \leq j$ and

$$
\frac{m}{j} \varphi^{*}\left(\frac{j}{m}\right) \geq \frac{m}{j-l} \varphi^{*}\left(\frac{j-l}{m}\right) .
$$

Hence

$$
\begin{aligned}
t^{j} & =t^{l} e^{-n \varphi^{*}(l / n)} t^{j-l} e^{n \varphi^{*}(l / n)} \geq e^{n \omega(t)-\log (t)}\left(\frac{e^{(m / j) \varphi^{*}(j / m)}}{e}\right)^{j-l} e^{m \varphi^{*}(l / m)} \\
& \geq e^{n \omega(t)-\log (t)} e^{m \varphi^{*}((j-l) / m)+m \varphi^{*}(l / m)} e^{-j} \geq e^{n \omega(t)-\log (t)} e^{-j} e^{2 m \varphi^{*}(j / 2 m)},
\end{aligned}
$$

the last inequality being a consequence of 1.4(1). The second statement of the lemma follows as the above inequality with $t=e^{(n / j) \varphi^{*}(j / n)}$.

Lemma 3.12. Let $\sigma(t)=t^{d}, 0<d<1$, and let $\omega$ be a weight function such that $\omega(t)=o(\sigma(t))$. Then there are $\lambda>0$ and a sequence $\left(j_{n}\right)$ of natural numbers such that

$$
\lambda \sigma\left(e^{(n / j) \varphi^{*}(j / n)}\right) \geq j \quad \text { for every } j \geq j_{n} .
$$

Proof. For every $n \in \mathbb{N}$ there is $A_{n}>0$ with $\omega(t) \leq A_{n}+n^{-1} \sigma(t)$ for all $t \geq 0$. Hence

$$
\frac{n}{j} \varphi_{\omega}^{*}\left(\frac{j}{n}\right) \geq-\frac{n A_{n}}{j}+\frac{1}{j} \varphi_{\sigma}^{*}(j) .
$$

We take $j_{n}$ satisfying $n A_{n} / j_{n} \leq 1$. Now the conclusion follows from the fact that $(1 / j) \varphi_{\sigma}^{*}(j)=(1 / d) \log (j / e d)$.

THEOREM 3.13. Let $\omega$ be a weight such that $\omega(t)=o\left(t^{d}\right), d \leq \varrho-\delta$, $d<1$. Let $a \in A S_{\varrho, \delta}^{m, \omega}(U)$ with associated pseudodifferential operator $A$ and let $\Omega$ be a relatively compact open subset of $U$. Then there are a pseudodifferential operator $P(x, D): \mathcal{D}_{(\omega)}(\Omega) \rightarrow \mathcal{E}_{(\omega)}(\Omega)$ and an $(\omega)$-smoothing operator $R: \mathcal{E}_{(\omega)}^{\prime}(\Omega) \rightarrow \mathcal{E}_{(\omega)}(\Omega)$ such that $A \varphi=P(x, D) \varphi+R \varphi$ for every $\varphi \in \mathcal{D}_{(\omega)}(\Omega)$. Moreover

$$
p(x, \xi) \sim \sum_{j=0}^{\infty} p_{j}(x, \xi), \quad \text { where } \quad p_{j}(x, \xi)=\sum_{|\alpha|=j} \frac{1}{\alpha !} D_{\xi}^{\alpha} \partial_{y}^{\alpha} a(x, y, \xi)_{\mid y=x} .
$$


Proof. For $p_{j}(x, \xi)$ as above, we take $p(x, \xi)$ as in Lemma 3.9 and $P:=$ $P(x, D)$. According to the previous lemmata, the operator $A-P: \mathcal{D}_{(\omega)}(\Omega)$ $\rightarrow \mathcal{E}_{(\omega)}(\Omega)$ can be represented as $A-P=\sum_{N=0}^{\infty} P_{N}$, where $P_{N}(u)(x)=$ $\int K_{N}(x, y) u(y) d y$ and the series is convergent in $L\left(\mathcal{D}_{(\omega)}(\Omega), \mathcal{D}_{(\omega)}^{\prime}(\Omega)\right)$. Here

$$
K_{N}(x, y):=\int\left(\varphi_{N}-\varphi_{N+1}\right)(\xi)\left(a(x, y, \xi)-\sum_{j=0}^{N} p_{j}(x, \xi)\right) e^{i(x-y) \xi} d \xi
$$

is a function in $\mathcal{E}_{(\omega)}(\Omega \times \Omega)$, as easily follows from the fact that $\varphi_{N}-\varphi_{N+1}$ has compact support. Hence, each $P_{N}$ is $(\omega)$-smoothing and our aim is to show that so is $\sum_{N=0}^{\infty} P_{N}$. To do this we need to obtain a different representation for this series. There is no loss of generality to assume that $\Omega$ is convex (in fact, in view of Theorem 2.17 we only have to show that every point $x \in \Omega$ admits a neighbourhood $W$ such that the kernel distribution of $A-P$ is an ultradifferentiable function of $(\omega)$-class in $W \times W$.)

Proceeding as in $[25,2.25]$ we get, for $N \geq 1, K_{N}=\sum_{|\alpha|=1}^{N} A_{\alpha}^{N}+R_{N}$, where

$$
A_{\alpha}^{N}(x, y):=\sum_{0 \neq \beta \leq \alpha} \frac{1}{\beta !(\alpha-\beta) !} \int e^{i(x-y) \xi} \sigma_{N, \alpha, \beta}(x, \xi) d \xi
$$

with

$$
\sigma_{N, \alpha, \beta}(x, \xi):=D_{\xi}^{\beta}\left(\varphi_{N}(\xi)-\varphi_{N+1}(\xi)\right) D_{\xi}^{\alpha-\beta} \partial_{y}^{\alpha} a(x, x, \xi),
$$

and

$$
R_{N}(x, y):=\sum_{|\alpha|=N+1} \sum_{\beta \leq \alpha} \frac{1}{\beta !(\alpha-\beta) !} \int e^{i(x-y) \xi} \tau_{N, \alpha, \beta}(x, y, \xi) d \xi
$$

with

$$
\tau_{N, \alpha, \beta}(x, y, \xi):=D_{\xi}^{\beta}\left(\varphi_{N}(\xi)-\varphi_{N+1}(\xi)\right) D_{\xi}^{\alpha-\beta} \omega_{\alpha}(x, y, \xi) .
$$

Here

$$
\omega_{\alpha}(x, y, \xi):=(N+1) \int_{0}^{1} \partial_{y}^{\alpha} a(x, x+t(y-x), \xi)(1-t)^{N} d t .
$$

On the other hand, $\sum_{r=1}^{N} \sum_{|\alpha|=1}^{r} A_{\alpha}^{r}=\sum_{j=1}^{N} I_{j}-W_{N}$, where

$$
I_{j}(x, y):=\sum_{|\alpha|=j} \sum_{0 \neq \beta \leq \alpha} \frac{1}{\beta !(\alpha-\beta) !} \int e^{i(x-y) \xi} D_{\xi}^{\beta} \varphi_{j}(\xi) D_{\xi}^{\alpha-\beta} \partial_{y}^{\alpha} a(x, x, \xi) d \xi
$$

and

$$
W_{N}(x, y):=\sum_{|\alpha|=1}^{N} \sum_{0 \neq \beta \leq \alpha} \frac{1}{\beta !(\alpha-\beta) !} \int e^{i(x-y) \xi} D_{\xi}^{\beta} \varphi_{N+1}(\xi) D_{\xi}^{\alpha-\beta} \partial_{y}^{\alpha} a(x, x, \xi) d \xi .
$$

Hence $\sum_{j=1}^{N} K_{j}=\sum_{j=1}^{N} I_{j}+\sum_{j=1}^{N} R_{j}-W_{N}$. To finish the proof we will show that $\sum_{j=1}^{\infty} R_{j}(x, y)$ and $\sum_{j=1}^{\infty} I_{j}(x, y)$ converge in $\mathcal{E}_{(\omega)}(\Omega \times \Omega)$ and 
that the sequence of operators defined by the kernels $\left(W_{N}\right)$ converges to the null operator as $N \rightarrow \infty$.

(a) Let $j_{n}<j \leq j_{n+1}$. Then

$$
\begin{aligned}
\left|D_{x}^{\mu} D_{y}^{\nu} I_{j}(x, y)\right| \leq & \sum_{|\alpha|=j} \sum_{0 \neq \beta \leq \alpha} \sum_{\gamma \leq \mu}\left(\begin{array}{l}
\mu \\
\gamma
\end{array}\right) \frac{1}{\beta !(\alpha-\beta) !} \\
& \times \int|\xi|^{|\gamma+\nu|}\left|D_{\xi}^{\beta} \varphi_{j}(\xi)\right|\left|D_{x}^{\mu-\gamma}\left(D_{\xi}^{\alpha-\beta} \partial_{y}^{\alpha} a(x, x, \xi)\right)\right| d \xi .
\end{aligned}
$$

We fix $k \in \mathbb{N}$ and we take $n \geq k$ and $l:=2 n$. If $D_{\xi}^{\beta} \varphi_{j}(\xi) \neq 0$ then

$$
2 \operatorname{Re}^{(n / j) \varphi^{*}(j / n)} \leq|\xi| \leq 3 \operatorname{Re}^{(n / j) \varphi^{*}(j / n)}
$$

and we see that

$$
\begin{aligned}
& \left|D_{x}^{\mu-\gamma} D_{\xi}^{\alpha-\beta} \partial_{y}^{\alpha} a(x, x, \xi)\right| \\
& \quad \leq C_{2 l}(\alpha-\beta) ! B^{|\alpha-\beta|} e^{(\varrho-\delta) 2 l \varphi^{*}(|\mu-\gamma+\alpha| / 2 l)} e^{m \omega(\xi)}|\xi|^{\delta(|\mu-\gamma|+|\alpha|)-\varrho|\alpha-\beta|} \\
& \quad \leq C_{2 l}(\alpha-\beta) ! B^{|\alpha-\beta|} e^{(\varrho-\delta)\left(k \varphi^{*}(|\mu-\gamma| / k)+l \varphi^{*}(j / l)\right)} e^{m \omega(\xi)}|\xi|^{\delta|\mu-\gamma|+\varrho|\beta|-(\varrho-\delta) j}
\end{aligned}
$$

by $1.3(1)$ and $k \leq l$. We now use the fact that $\left|D_{\xi}^{\beta} \varphi_{j}(\xi)\right| \leq C(D /|\xi|)^{|\beta|} j^{|\beta|+1}$ and $|\xi|^{\delta|\mu-\gamma|} e^{-\delta k \varphi^{*}(|\mu-\gamma| / k)} \leq e^{\delta k \omega(\xi)}$. Moreover $e^{-(n-1) \omega(\xi / 3 R)} e^{n \varphi^{*}(j / n)} \geq$ $e^{2 n \varphi^{*}(j / 2 n)}$ (see Lemma 3.11) and $|\xi|^{|\nu+\gamma|} \leq e^{k \varphi^{*}(|\nu+\gamma| / k)+k \omega(\xi)}$, thus

$$
\begin{aligned}
\left|D_{x}^{\mu} D_{y}^{\nu} I_{j}(x, y)\right| & \leq \sum_{|\alpha|=j} \frac{C_{4 n} B^{j} e^{k \varphi^{*}(|\mu+\nu| / k)}}{(2 R)^{(\varrho-\delta) j}} \\
& \times \sum_{\substack{0 \neq \beta \leq \alpha \\
\gamma \leq \mu}}\left(\begin{array}{c}
\mu \\
\gamma
\end{array}\right) \frac{1}{\beta !} C D^{|\beta|} j^{|\beta|+1} \int e^{(2 k+m) \omega(\xi)-(n-1)(\varrho-\delta) \omega(\xi / 3 R)} d \xi .
\end{aligned}
$$

Given $k$ we can select $n$ large enough in order to ensure that the integral above is less than 1 . Then, for $j_{n}<j \leq j_{n+1}$, we obtain

$$
\left|D_{x}^{\mu} D_{y}^{\nu} I_{j}(x, y)\right| \leq C C_{4 n} j^{p+1} B^{j} \frac{e^{D j p^{2}}}{(2 R)^{(\varrho-\delta) j}} e^{|\mu|+k \varphi^{*}(|\mu+\nu| / k)} .
$$

Proceeding as in the proof of 3.7 we can select the sequence $\left(j_{n}\right)$ and the constant $R>0$ in order to guarantee the convergence of $\sum I_{j}(x, y)$ in $\mathcal{E}_{(\omega)}(\Omega \times \Omega)$.

(b) With a similar argument it is possible to prove that $\sum_{j=1}^{\infty} R_{j}(x, y)$ converges in $\mathcal{E}_{(\omega)}(\Omega \times \Omega)$ for a suitable choice of $\left(j_{n}\right)$ and $R>0$. In fact, we recall that

$$
R_{j}(x, y)=\sum_{|\alpha|=j+1} \sum_{\beta \leq \alpha} \frac{1}{\beta !(\alpha-\beta) !} \int e^{i(x-y) \xi} \tau_{j, \alpha, \beta}(x, y, \xi) d \xi .
$$


Hence

$$
\begin{aligned}
\left|D_{x}^{\mu} D_{y}^{\nu} R_{j}(x, y)\right| \leq & \sum_{|\alpha|=j+1} \sum_{\beta \leq \alpha} \sum_{\gamma \leq \mu} \sum_{r \leq \nu}\left(\begin{array}{c}
\mu \\
\gamma
\end{array}\right)\left(\begin{array}{c}
\nu \\
r
\end{array}\right) \frac{1}{\beta !(\alpha-\beta) !} \\
& \times \int|\xi|^{|\gamma+r|}\left|D_{x}^{\mu-\gamma} D_{y}^{\nu-r} \tau_{j, \alpha, \beta}(x, y, \xi)\right| d \xi .
\end{aligned}
$$

Now, for a fixed $k \in \mathbb{N}$ we take $n \geq k$ and $l=2 n+2$. Then the quantity $\left|D_{x}^{\mu-\gamma} D_{y}^{\nu-r} \tau_{j, \alpha, \beta}(x, y, \xi)\right|$ is less than or equal to the product of

$$
\left|D_{\xi}^{\beta}\left(\varphi_{j}-\varphi_{j+1}\right)(\xi)\right|(j+1)
$$

by

$$
\sum_{s \leq \mu-\gamma}\left(\begin{array}{c}
\mu-\gamma \\
s
\end{array}\right) \int_{0}^{1}\left|D_{y}^{\alpha+\nu-r} D_{x}^{s} D_{y}^{\mu-\gamma-s} D_{\xi}^{\alpha-\beta} a(x, x+t(y-x), \xi)\right| d t .
$$

The above integral is dominated by

$$
C_{2 l} B^{|\alpha-\beta|}(\alpha-\beta) ! \frac{e^{(\varrho-\delta)\left(k \varphi^{*}(|\mu+\nu-r-\gamma| / k)+l \varphi^{*}(j+1 / l)\right)}}{|\xi|^{\varrho|\alpha-\beta|-\delta|\mu+\nu+\alpha-r-\gamma|}} e^{m \omega(\xi)} .
$$

Having in mind

$$
|\xi|^{\delta|\mu+\nu-r-\gamma|} e^{-\delta k \varphi^{*}(|\mu+\nu-r-\gamma| / k)} \leq e^{\delta k \omega(\xi)}
$$

(Lemma 1.4) and

$$
|\xi|^{|\gamma+r|} e^{\varrho k \varphi^{*}(|\mu+\nu-r-\gamma| / k)} \leq e^{k \omega(\xi)} e^{k \varphi^{*}(|\mu+\nu| / k)}
$$

(1.3 and 1.4) we conclude that

$$
|\xi|^{|\gamma+r|}\left|D_{x}^{\mu-\gamma} D_{y}^{\nu-r} \tau_{j, \alpha, \beta}(x, y, \xi)\right|
$$

is less than or equal to the product of $\left|D_{\xi}^{\beta}\left(\varphi_{j}-\varphi_{j+1}\right)(\xi)\right|$ by

$$
\begin{aligned}
\frac{e^{(k+m+\delta k) \omega(\xi)}}{|\xi|^{\varrho(j+1-|\beta|)-\delta(j+1)}} 2^{|\mu-\gamma|} B^{j+1-|\beta|} C_{2 l}( & \alpha-\beta) !(j+1) \\
& \times e^{k \varphi^{*}(|\mu+\nu| / k)} e^{(\varrho-\delta) l \varphi^{*}((j+1) / l)} .
\end{aligned}
$$

An application of the previous lemmata shows that we can select $n$ in such a way that the above bound is less than

$$
C D^{|\beta|}(j+1)^{|\beta|} C_{2 l} \frac{e^{-\omega(\xi)}}{R^{(\varrho-\delta)(j+1)}} 2^{|\mu-\gamma|} B^{j+1-|\beta|}(\alpha-\beta) !(j+1)^{2} e^{k \varphi^{*}(|\mu+\nu| / k)} .
$$

Hence

$$
\left|D_{x}^{\mu} D_{y}^{\nu} R_{j}(x, y)\right| \leq\left(\frac{e^{p^{2} D} B}{R^{\varrho-\delta}}\right)^{j+1}(j+1)^{p+2} C_{2 l} 2^{2|\mu+\nu|} e^{k \varphi^{*}(|\mu+\nu| / k)} .
$$

It follows that, after choosing $\left(j_{n}\right)$ and $R$ in the proper way, the series $\sum R_{j}$ converges in $\mathcal{E}_{(\omega)}(\Omega \times \Omega)$. 
(c) Let $T_{N}: \mathcal{D}_{(\omega)}(\Omega) \rightarrow \mathcal{D}_{(\omega)}^{\prime}(\Omega)$ be the operator with kernel $W_{N}$. Since $\sum_{N=0}^{\infty} P_{N}$ converges in $L\left(\mathcal{D}_{(\omega)}(\Omega), \mathcal{D}_{(\omega)}^{\prime}(\Omega)\right)$, we deduce from (a) and (b) that $\left(T_{N}\right)$ converges to an operator $T: \mathcal{D}_{(\omega)}(\Omega) \rightarrow \mathcal{D}_{(\omega)}^{\prime}(\Omega)$ in $L\left(\mathcal{D}_{(\omega)}(\Omega)\right.$, $\left.\mathcal{D}_{(\omega)}^{\prime}(\Omega)\right)$. In order to show that $T=0$ it is sufficient to prove that $T$ vanishes on the dense subset $\mathcal{D}_{(\sigma)}(\Omega), \sigma(t):=t^{d}$. To do this, we fix $N \in \mathbb{N}$ with $j_{n}<N+1 \leq j_{n+1}$, and we put $a_{N}:=\operatorname{Re}^{\frac{n}{N+1} \varphi^{*}\left(\frac{N+1}{n}\right)}$. Then $D_{\xi}^{\beta} \varphi_{N+1}(\xi) \neq 0$ implies that $2 a_{N} \leq|\xi| \leq 3 a_{N}$. For every $u \in \mathcal{D}_{(\sigma)}(\Omega)$ we have

$$
\begin{aligned}
\left|T_{N}(u)(x)\right| & \leq \sum_{|\alpha|=1}^{N} \sum_{0 \neq \beta \leq \alpha} \frac{1}{\beta !(\alpha-\beta) !}\left|\int D_{\xi}^{\beta} \varphi_{N+1}(\xi) D_{\xi}^{\alpha-\beta} \partial_{y}^{\alpha} a(x, x, \xi) \widehat{u}(\xi) d \xi\right| \\
& \leq \sum_{|\alpha|=1}^{N} \sum_{0 \neq \beta \leq \alpha} \frac{D^{|\beta|}(N+1)^{|\beta|+1}}{\beta !} \frac{B^{|\alpha-\beta|} C_{n}}{(2 R)^{(\varrho-\delta)|\alpha|}} \int_{|\xi| \geq 2 a_{N}} e^{m \omega(\xi)}|\widehat{u}(\xi)| d \xi .
\end{aligned}
$$

Let $\lambda$ be given as in Lemma 3.12. Then $\lambda \sigma\left(a_{N}\right) \geq N+1$. For every $u \in$ $\mathcal{D}_{(\sigma)}(\Omega)$ we have $|\widehat{u}(\xi)| \leq e^{-\left(m+\lambda p^{2}+1\right) \sigma(\xi)}$ for $|\xi|$ large enough. Since $\log (t)=$ $o(\sigma(t))$ we obtain

$$
\int_{|\xi| \geq 2 a_{N}} e^{m \omega(\xi)}|\widehat{u}(\xi)| d \xi \leq \frac{e^{-\lambda p^{2} \sigma\left(2 a_{N}\right)}}{\left(2 a_{N}\right)^{2}}<\frac{1}{2 a_{N} e^{p^{2}(N+1)}}
$$

for $N$ large enough. By Lemma 3.10, we can assume that $j / e^{(n / j) \varphi^{*}(j / n)}$ $\leq 1 / 2^{n}$ for $j \geq j_{n}$. Consequently, since $\sum_{\beta \leq \alpha}(N+1)^{|\beta|} / \beta ! \leq e^{p^{2}(N+1)}$, we get

$$
\left|T_{N}(u)(x)\right| \leq \frac{1}{2^{n}} \sum_{|\alpha|=1}^{N}\left(\frac{B D}{2 R^{\varrho-\delta}}\right)^{|\alpha|} \frac{N+1}{a_{N}} \frac{C_{n}}{2 a_{N}} \leq \frac{1}{2^{n}} \sum_{j=1}^{N}\left(\frac{p D B}{R^{\varrho-\delta}}\right)^{j},
$$

from which we deduce that $T_{N}(u)(x)$ converges to 0 uniformly on $x \in \Omega$ as $N$ goes to infinity.

In order to compose pseudodifferential operators it is useful to consider operations with formal sums.

Proposition 3.14. Let $P(x, D)$ be the operator associated to $p(x, \xi) \in$ $A S_{\varrho, \delta}^{m, \omega}(U)$ and let $\Omega$ be a relatively compact open subset of $U$. Then the transposed operator (restricted to $\mathcal{D}_{(\omega)}(\Omega)$ ) can be decomposed as $P(x, D)^{t}=$ $Q(x, D)+R$, where $R$ is $(\omega)$-smoothing and $Q(x, D)$ is defined by a symbol $q(x, \xi) \sim \sum q_{j}$, and we have $q_{j}(x, \xi):=\sum_{|\alpha|=j}(1 / \alpha !) \partial_{\xi}^{\alpha} D_{x}^{\alpha} p(x,-\xi)$.

Proof. We already know that $P(x, D)^{t}$ is associated to $p(y,-\xi)$. Then apply 3.13. 
Given $\sum p_{j} \in F A S_{o, \delta}^{m, \omega}(\Omega)$, standard calculations ([25]) and the properties of $\varphi^{*}$ prove that $\sum q_{j}$, where $q_{j}(x, \xi):=\sum_{|\alpha|+h=j}(1 / \alpha !) \partial_{\xi}^{\alpha} D_{x}^{\alpha} p_{h}$, is a formal sum.

Analogously, if $\sum p_{j} \in F A S_{\varrho, \delta}^{m_{1}, \omega}(\Omega)$ and $\sum q_{j} \in F A S_{\varrho, \delta}^{m_{2}}$, one can prove that $\sum r_{j} \in F A S_{\varrho, \delta}^{m_{1}+m_{2}, \omega}(\Omega)$, where $r_{j}(x, \xi)=\sum_{|\alpha|+k+h=j}(1 / \alpha !) \partial_{\xi}^{\alpha} p_{h} D_{x}^{\alpha} q_{j}$ is a formal sum.

Definition 3.15. (1) For $\sum p_{j} \in F A S_{\varrho, \delta}^{m, \omega}(\Omega)$ we define $\left(\sum p_{j}\right)^{t}$ as the formal sum $\sum_{j} q_{j}$, where $q_{j}$ is as before.

(2) For $\sum p_{j} \in F A S_{\varrho, \delta}^{m_{1}, \omega}(\Omega)$ and $\sum q_{j} \in F A S_{\varrho, \delta}^{m_{2}}$ we define $\left(\sum p_{j}\right) \circ$ $\left(\sum q_{j}\right)=\sum r_{j}$, where $r_{j}$ is as above.

The following two results are straightforward, therefore we omit their proof $([25])$.

Proposition 3.16. (1) $\left(\left(\sum p_{j}\right)^{t}\right)^{t} \sim \sum p_{j}$.

(2) If $\sum p_{j} \sim \sum p_{j}^{\prime}$ and $\sum q_{j} \sim \sum q_{j}^{\prime}$, then $\left(\sum p_{j}\right) \circ\left(\sum q_{j}\right) \sim\left(\sum p_{j}^{\prime}\right) \circ$ $\left(\sum q_{j}^{\prime}\right)$.

Lemma 3.17. Let $\Omega \subset \mathbb{R}^{p}$ be an open bounded set, and let $p(x, \xi), q(x, \xi)$ $\in A S_{\varrho, \delta}^{m, \omega}(\Omega)$. Assume $b(x, \xi) \in A S_{\varrho, \delta}^{m, \omega}(\Omega)$ satisfies $b(x, \xi) \sim q^{t}(x,-\xi)$ and $r(x, \xi) \in A S_{\varrho, \delta}^{2 m, \omega}(\Omega)$ is equivalent to $\left.\sum_{j} \sum_{|\alpha|=j} \frac{1}{\alpha !} \partial_{\xi}^{\alpha} D_{y}^{\alpha}(p(x, \xi) b(y, \xi))\right|_{y=x}$. Then $r(x, \xi) \sim p(x, \xi) \circ q(x, \xi)$.

Theorem 3.18. Let $p(x, \xi), q(x, \xi) \in A S_{o, \delta}^{m, \omega}(U)$ and let $\Omega$ be an open set which is relatively compact in $U$. Denote by $P$ and $Q$ the corresponding pseudodifferential operators and assume that either $P$ or $Q$ is properly supported. Then $P \circ Q: \mathcal{D}_{(\omega)}(\Omega) \rightarrow \mathcal{E}_{(\omega)}(\Omega)$ coincides, modulo an $(\omega)$-smoothing operator, with the pseudodiferential operator associated to $(2 \pi)^{p}(p(x, \xi) \circ q(x, \xi))$.

Proof. Assume that $P$ is properly supported. We take $\Omega_{1}$ relatively compact and open in $U$ containing $\bar{\Omega}$.

We know that $Q=\left(Q^{t}\right)^{t}$ and that $Q^{t}$ is given by $q(y,-\xi)$. Therefore $Q^{t}=Q^{\prime}+T^{\prime}$ on $\Omega_{1}$, where $T^{\prime}$ is $(\omega)$-smoothing and $Q^{\prime}$ is given by a symbol $q^{\prime}$ on $\Omega_{1}$, which is equivalent to $q^{t}$. Since the class of $(\omega)$ smoothing operators is closed under taking transposes on $\Omega_{1}, Q$ coincides, modulo some $(\omega)$-smoothing operator, with the operator $Q_{1}$ associated to $b(y, \xi):=q^{\prime}(y,-\xi) \sim q^{t}(y,-\xi)$. As composition of $P$ with any $(\omega)$-smoothing operator is again $(\omega)$-smoothing, $P \circ Q-P \circ Q_{1}$ is $(\omega)$-smoothing.

Given $f \in \mathcal{D}_{(\omega)}(\Omega)$ we have $Q_{1} f \in \mathcal{D}_{L_{1},(\omega)}\left(\mathbb{R}^{p}\right)$ (Prop. 2.16), therefore $P\left(Q_{1} f\right)(x)=\int p(x, \xi) \widehat{Q_{1} f}(\xi) d \xi$ (Prop. 2.4). But, from 2.16, $Q_{1} f(x)=$ $\widehat{I}(-x)$, therefore $\widehat{Q_{1} f}(\xi)=(2 \pi)^{p} I(\xi)$. That is, $P \circ Q_{1}$ is the pseudodifferential operator associated to $a(x, y, \xi)=(2 \pi)^{p} p(x, \xi) b(y, \xi)$. We apply 3.13 and 3.17 to conclude. 
Acknowledgements. The authors are grateful to the referee for the careful reading of the paper and for some valuable remarks and corrections.

\section{References}

[1] J. J. Betancor, C. Fernández and A. Galbis, Beurling ultradistributions of $L_{p}$-growth, J. Math. Anal. Appl. 279 (2003), 246-265.

[2] G. Björk, Linear partial differential operators and generalized distributions, Ark. Mat. 6 (1965), 351-407.

[3] J. Bonet, C. Fernández and R. Meise, Characterization of the w-hypoelliptic convolution operators on ultradistributions, Ann. Acad. Sci. Fenn. Math. 25 (2000), 261-284.

[4] -, - , -, Operators of solution for convolution equations, Note Mat. 17 (1997), $1-12$.

[5] L. Boutet de Monvel, Opérateurs pseudo-différentiels analytiques et opérateurs d'ordre infini, Ann. Inst. Fourier 22 (1972), no. 3, 229-268.

[6] L. Boutet de Monvel and P. Kree, Pseudo-differential operators and Gevrey classes, ibid. 17 (1967), no. 1, 295-323.

[7] R. W. Braun, An extension of Komatsu's second structure theorem for ultradistributions, J. Fac. Sci. Univ. Tokyo Sect. IA Math. 40 (1993), 411-417.

[8] R. W. Braun, R. Meise and B. A. Taylor, Ultradifferentiable functions and Fourier analysis, Results Math. 17 (1990), 206-237.

[9] A. P. Calderón, Lecture Notes on Pseudo-Differential Operators and Elliptic Boundary Value Problems, Consejo Nacional de Investigaciones Científicas y Técnicas, Instituto Argentino de Matemática, Buenos Aires, 1976.

[10] C. C. Chou, La transformation de Fourier complexe et l'équation de convolution, Lecture Notes in Math. 325, Springer, Berlin, 1973.

[11] C. Fernández, A. Galbis and M. C. Gómez-Collado, Elliptic convolution operators on non-quasianalytic classes, Arch. Math. (Basel) 76 (2001), 133-140.

[12] M. C. Gómez-Collado, Ultradistribuciones casi-periódicas y operadores de convolucion elipticos, $\mathrm{PhD}$ thesis, Univ. de Valencia, 2000.

[13] A. Grigis and J. A. Sjöstrand, Microlocal Analysis for Differential Operators. An Introduction, London Math. Soc. Lecture Note Ser. 196, Cambridge Univ. Press, Cambridge, 1994.

[14] L. Hörmander, Pseudo-differential operators, Comm. Pure Appl. Math. 18 (1965), 129-209.

[15] S. Hashimoto, T. Matsuzawa and Y. Morimoto, Opérateurs pseudo-différentiels et classes de Gevrey, Comm. Partial Differential Equations 8 (1983), 1277-1289.

[16] H. Komatsu, Ultradistributions I. Structure theorems and a characterization, J. Fac. Sci. Univ. Tokyo Sec. IA 20 (1973), 25-105.

[17] - An Introduction to the Theory of Generalized Functions, Dept. of Math., Science Univ. of Tokyo, 2000.

[18] J. J. Kohn and L. Nirenberg, An algebra of pseudo-differential operators, Comm. Pure Appl. Math. 18 (1965), 269-305.

[19] M. Langenbruch, Continuation of Gevrey regularity for solutions of partial differential operators, in: Functional Analysis (Trier, 1994), de Gruyter, 1996, 249-280.

[20] O. Liess and L. Rodino, Inhomogeneous Gevrey classes and related pseudodifferential operators, Boll. Un. Mat. Ital. C (6) 3 (1984), 233-323. 
[21] W. Matsumoto, Theory of pseudo-differential operators of ultradifferentiable class, J. Math. Kyoto Univ. 27 (1987), 453-500.

[22] R. Meise and B. A. Taylor, Whitney's extension theorem for ultradifferentiable functions of Beurling type, Ark. Mat. 26 (1988), 265-287.

[23] L. Rodino, Linear Partial Differential Operators in Gevrey Spaces, World Sci., 1993.

[24] F. Treves, Introduction to Pseudodifferential and Fourier Integral Operators I, Plenum Press, New York, 1980.

[25] L. Zanghirati, Pseudodifferential operators of infinite order and Gevrey classes, Ann. Univ. Ferrara Sez. VII 31 (1985), 197-219.

C. Fernández and A. Galbis

Departamento de Análisis Matemático

Universidad de Valencia

Doctor Moliner 50

46100 Burjasot (Valencia), Spain

E-mail: Carmen.Fdez-Rosell@uv.es

Antonio.Galbis@uv.es
D. Jornet

Departamento de Matemática Aplicada

ETSI Telecomunicación Universidad Politécnica de Valencia

E-46071 Valencia, Spain E-mail: djornet@mat.upv.es

Received February 7, 2003

Revised version October 1, 2003 